\title{
SMALL-SCALE STRUCTURES IN PLANETARY NEBULAE
}

A Thesis presented to

the Faculty of the Graduate School

at the University of Missouri

In Partial Fulfillment

of the Requirements for the Degree

Doctor of Philosophy

by

SEAN P. BALDRIDGE

Dr. Angela Speck, Thesis Supervisor

DEC 2017 
The undersigned, appointed by the Dean of the Graduate School, have examined the dissertation entitled:

\section{SMALL-SCALE STRUCTURES IN PLANETARY NEBULAE}

presented by Sean P. Baldridge, a candidate for the degree of Doctor of Philosophy and hereby certify that, in their opinion, it is worthy of acceptance.

Dr. Angela Speck

Dr. Haojing Yan

Dr. Aigen Li

Dr. Rainer Glaser

Dr. Yicheng Guo 


\section{ACKNOWLEDGMENTS}

First and foremost, I would like to thank my adviser, Dr. Angela Speck, for her countless contributions toward my research these past 6 years. I would also like to thank Dr. Mikako Matsuura and Dr. George Jacoby for graciously allowing me to use their Dumbbell nebula images and spectra in addition to helpful comments on my writing. I am also grateful to the powers that be at MU for buying into the WIYN Consortium, which made it possible to obtain the necessary images of NGC 2392. Dr. Alan (Elric) Whittington, thank you for making me aware of the need to change the common name of NGC 2392. Finally, I would like to thank all those who aided in obtaining WIYN data for my research: Lisa Shepard, Eric Cropp, Marat Musin, and all the OAs at the WIYN telescope.

I want to give special thanks to Dr. Bradley Schaefer, whose introductory astronomy course brought to life a passion for astronomy and astrophysics which gave me a direction to pursue in my physics career. 


\section{TABLE OF CONTENTS}

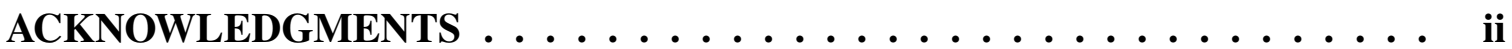

LIST OF TABLES .........................

LIST OF FIGURES .......................... vi

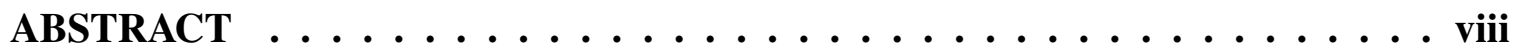

\section{CHAPTER}

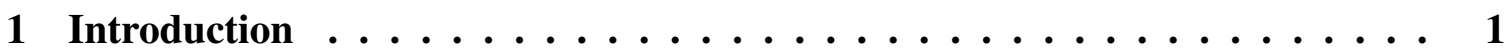

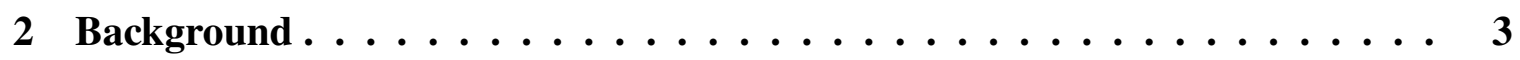

2.1 Stellar Mass Loss . . . . . . . . . . . . . . . . . . . . . . . . . . . 3

2.1.1 Stellar Evolution . . . . . . . . . . . . . . . . 3

2.2 Planetary Nebula: A Star's Dying Breath . . . . . . . . . . . . . 6

2.2.1 Morphology .................. 8

2.2.2 Small-Scale Structures . . . . . . . . . . . . . . . . 10

2.2.3 Molecular Hydrogen . . . . . . . . . . . . . . . . . . . 14

3 The Dumbbell Nebula . . . . . . . . . . . . . . . . . 19

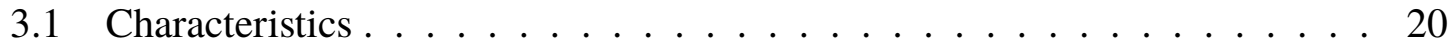

3.2 Observations and data reduction ................. 21

3.2.1 Near-IR (Subaru) . . . . . . . . . . . . . . . 21

3.2.2 Visible $(\mathrm{WIYN}) \ldots \ldots \ldots 24$

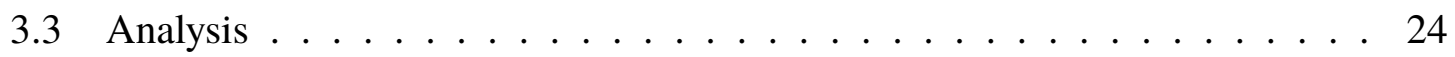


3.3.1 Image Analysis . . . . . . . . . . . . . . . 24

3.3 .2 Spectral Analysis . . . . . . . . . . . . . . . 28

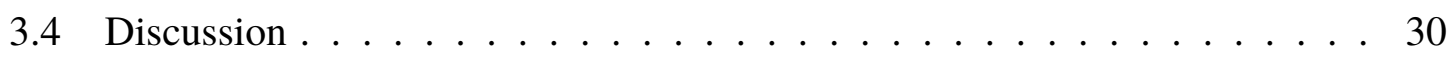

3.4.1 Comparison to other $\mathrm{PNe} \ldots \ldots \ldots . \ldots 30$

3.4.2 Knot and $\mathrm{H}_{2}$ Formation . . . . . . . . . . . . . . 33

3.4.3 Excitation Temperatures ................. 34

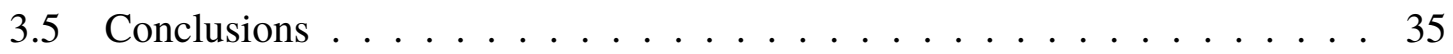

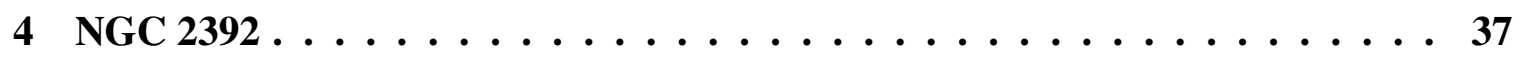

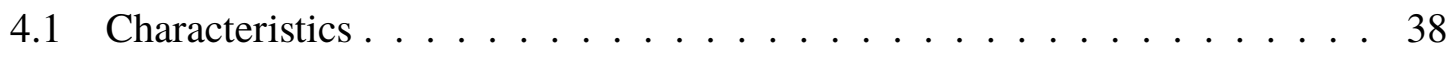

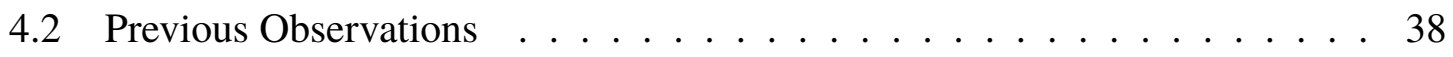

4.3 Observations .......................... 41

4.4 Image and Spectral Analysis . . . . . . . . . . . . . . 41

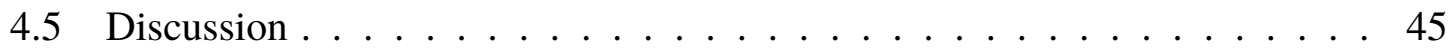

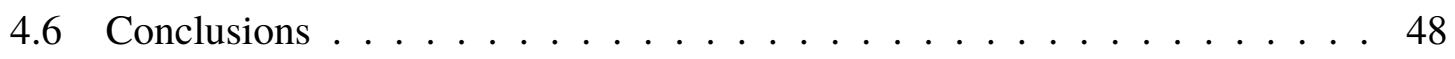

5 Summary and concluding remarks ................... 49 APPENDIX

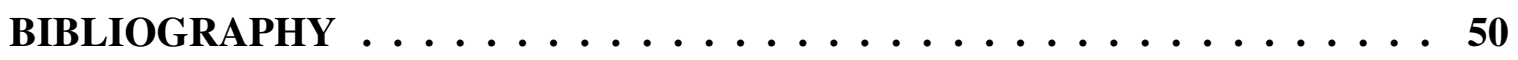

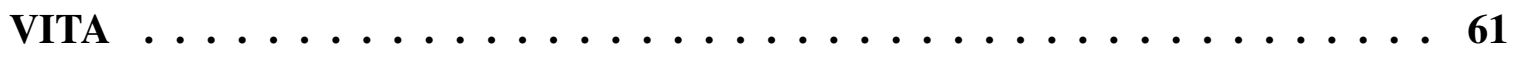




\section{LIST OF TABLES}

Table

Page

2.1 List of PNe with observable knots and whether they exhibit any $\mathrm{H}_{2}$ emission . . . 18

3.1 Line ratios and excitation temperatures of $\mathrm{H}_{2}$ Near-IR lines. . . . . . . . 35 


\section{LIST OF FIGURES}

$\begin{array}{ll}\text { Figure } & \text { Page }\end{array}$

2.1 The Helix Nebula. Left: Infrared image of Helix nebula. Credit: ESO/VISTA/J. Emerson; Right: HST image of cometary knots. Credit: NASA,C.R. O’Dell; . . . 8

2.2 The Ring Nebula. Left: HST Visible. Image credit: The Hubble Heritage Team (AURA/STScI/NASA); RIGHT: Ground based $\mathrm{H}_{2}$ image $(2.12 \mu \mathrm{m})$. Image credit: Speck et al. (2003). . . . . . . . . . . . . . . . . . . . . 14

3.1 Color composite image of the Dumbbell nebula. Credit: George Jacoby, WIYN telescope . . . . . . . . . . . . . . . . . 19

$3.2 \mathrm{H}_{2}$ image of Dumbbell Nebula taken by MOIRCS on Subaru . . . . . . . 22

3.3 Map of slit locations used on MOIRCS; The box represents the zoomed region in Figure $3.9 \ldots \ldots \ldots . \ldots \ldots$

3.4 (a) [O I] image of Dumbbell Nebula taken by WIYN; (b) $\mathrm{H} \alpha$ image of the Dumbbell taken by WIYN; (c) [O III] image of the Dumbbell taken by

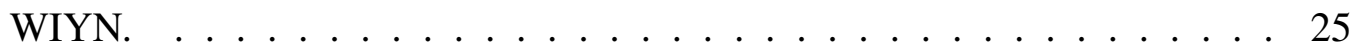

3.5 Superposition of the Subaru $\mathrm{H}_{2}$ image (blue) and WIYN $\mathrm{H} \alpha$ image (red). Arrow indicates the region where $\mathrm{H}_{2}$ dominates at the edge of the visible

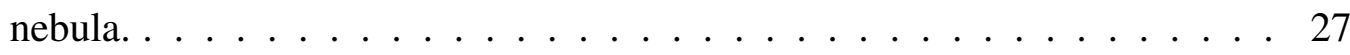


3.6 Spectrum 1 (diffuse), $x$-axis is wavelength measured in $\mu \mathrm{m}, y$-axis is flux (not calibrated) . . . . . . . . . . . . . . . 28

3.7 Spectrum 2 (knot), $x$-axis is wavelength measured in $\mu \mathrm{m}, y$-axis is flux (not

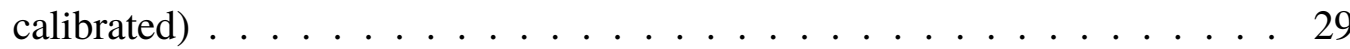

3.8 Spectrum 3 (knot), $x$-axis is wavelength measured in $\mu \mathrm{m}, y$-axis is flux (not

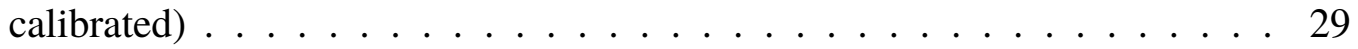

3.9 Top: Middle region of the Helix nebula in $\mathrm{H}_{2}$ (Matsuura 2009). Bottom: Region of Dumbbell nebula indicated in Figure 3.3. . . . . . . . . . . . . 31

4.1 HST image of NGC 2392. Credit: Andrew Fruchter (STScI, NASA) . . . . 37

4.2 Narrow band images of NGC 2392 around $2 \mu \mathrm{m}$ taken at WIYN (top) and visible narrow band HST images (bottom). . . . . . . . . . . . . . . . . 42

4.3 K-band spectrum obtained by H99 of the inner region of NGC 2392 . . . . 43

4.4 Flux calibrated images of $\mathrm{H}_{2}$ (left) and Brg45 (right). Scale bar is in units

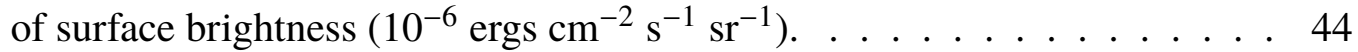

$4.5 \quad 8-12 \mu$ m spectrum of NGC 2392, Spitzer. Inset: Map of slit positions . . . 45 


\begin{abstract}
Two planetary nebulae (PNe) were studied in order to investigate small scale molecular structures in planetary nebulae, the survival of which affects the structure and composition of the interstellar medium (ISM) from which the next generation of stars is born. Molecular hydrogen $\left(\mathrm{H}_{2}\right)$ was used a tracer for molecular emission and its excitation properties used as a probe of shock physics. Previous studies of several planetary nebulae have indicated a relationship between molecular hydrogen and cometary knots as a shielding mechanism allowing for continued survival and formation of the molecule. Our first PN studied, the Dumbbell nebula (NGC 6853), revealed not only $\mathrm{H}_{2}$ bearing knots (similar to the Helix and Ring nebulae), but also regions of $\mathrm{H}_{2}$ emission which were poorly sculpted or unstructured diffuse regions, unlike previously studied nebulae. The second PN, NGC 2392 has well documented cometary knots, however we find no detection of molecular hydrogen emission from the knots or otherwise making it an outlier among cometary knot bearing planetary nebulae. Both nebula studied in this research challenge current models of $\mathrm{H}_{2}$ bearing small scale structures in PNe and indicate a need to expand our rather small sample of such objects so that we may begin to understand the processes behind $\mathrm{H}_{2}$ formation, excitation, and its role in cometary knots of the PN as a whole.
\end{abstract}




\section{Chapter 1}

\section{Introduction}

The end of a star's life is typically marked by phenomena which return a portion of the star's matter back into the interstellar medium (ISM) from which new stars are born. The progenitor mass of the star determines which of two paths the star may take; stars of high mass will undergo violent deaths through type II supernovae while stars of intermediate mass will gently shed their outer layers on timescales of $10^{5}-10^{6}$ years, the final stage being seen as a planetary nebula $(\mathrm{PNe})$. The planetary nebula is the circumstellar shell of material surrounding the hot, shrinking stellar remnant which is host to a myriad of chemistries. The unique environment a PN provides allows them to serve as laboratories for investigating atomic, molecular, dust, and plasma physics which have applications to diverse other astrophysical environments. The formation, destruction, and survival of molecules are of particular interest since molecules play a pivotal role in the formation of new stars. The molecular clouds (from which new stars and planets are born) are expected to contain molecules formed in situ in the ISM using dust grains as catalysts. While molecules are formed around dying stars, they are expected to be destroyed by the high energy photons in 
interstellar space or as dying stars evolve into planetary nebulae (or supernova). However, the discovery of vast quantities of molecular gas $\left(\right.$ mostly $\left.\mathrm{H}_{2}\right)$ within the ionized regions of PNe suggests that some of the molecules created in stellar death can survive harsh environments and may seed the ISM directly which would affect the formation of subsequent stars and planets.

The survival of molecular material during the planetary nebula stage can be attributed to the shielding effect of small density-enhanced regions, or small-scale structures. The term 'small' is in reference to the entire nebula, while the small-scale structures themselves are typically on the order of a solar system in size. The most famous of these small-scale structures are the cometary knots seen in the Helix nebula. Determining whether these small-scale structures, or globules ${ }^{1}$, can survive the harsh environment of a PN and enter the ISM intact would have implications in the evolution of the Universe.

In order to understand how the globules evolve in time, we must explore their relationship with varying stages of stellar evolution, the role of molecular formation and shielding, and predict their possible survival. Molecular hydrogen is used as a tracer for molecular material and the properties of its emission used as a probe into the physical environment of the globules.

The work presented here will explore the origins of cometary knots in PNe in addition to determining their link to molecular hydrogen, if any. In the next chapter, we will discuss the relevant background. The Dumbbell nebula and NGC 2392 have been studied in depth for this work and are discussed in chapters 3 and 4, respectively. A summary and conclusion can be found in chapter 5 .

\footnotetext{
${ }^{1}$ the term "globule" is a catch-all for knots, clumps and other small-scale structures
} 


\section{Chapter 2}

\section{Background}

\subsection{Stellar Mass Loss}

\subsubsection{Stellar Evolution}

The stars that eventually become PNe start their lives as low- to intermediate-mass stars (LIMS; $0.8 \mathrm{M}_{\odot}, \lesssim \mathrm{M}_{\mathrm{MS}}{ }^{1} \lesssim 8 \mathrm{M}_{\odot}$, (Kwok, 2004). These stars eventually evolve off the main sequence to the Red Giant Branch and subsequently become asymptotic giant branch (AGB) stars, ending their lives as cooling white dwarfs. Between the AGB phase and the white dwarf phase, some of these stars become PNe as the shrinking, heating central stellar core subsequently illuminates material ejected by the AGB star. However, precisely which AGB stars go through the PNe phase is not clear (e.g., Sahai (2009) and references therein).

\footnotetext{
${ }^{1} \mathrm{M}_{\mathrm{MS}}=$ stellar main-sequence mass
} 


\section{Asymptotic Giant Branch (AGB) stars}

AGB stars are luminous $\left(L_{\star} \approx 10^{4} \mathrm{~L}_{\odot}\right), \operatorname{cool}\left(T_{\mathrm{eff}} \approx 3000 \mathrm{~K}\right)$ giants $\left(R_{\star} \approx 1 \mathrm{AU}\right)$, which lose mass copiously. A combination of stellar pulsation and low surface gravity leads to atmospheric gases escaping from their parent stars. Pulsations levitate atmospheric material allowing it to achieve an altitude where cooling temperatures allow molecules to form (see e.g. Alexander and Ferguson (1994), followed by formation of microscopic solid particles (dust grains). As a result, dusty molecular circumstellar envelopes surround AGB stars. Once dust is formed, radiation pressure becomes very important. The dust grains tap into the tremendous luminosity power of the star. As the dust grains are driven outwards by radiation pressure, they drag along the surrounding molecular gas (e.g. Hofner and Dorfi (1997).

AGB stars lose mass at high rates $\left(10^{-7}\right.$ to a few times $\left.10^{-4} \mathrm{M}_{\odot} / \mathrm{yr}\right)$, and generally the mass-loss rate, $\dot{M}$, increases over time as the star evolves. Continued mass loss causes the dust shell to increase in depth both optically and geometrically as mass-loss rate increases. The AGB phase ends in an episode of extremely high mass loss $\left(\dot{M}>10^{-5} \mathrm{M}_{\odot} \mathrm{yr}^{-1}\right)$, the superwind (SW) phase (Iben and Renzini, 1983; Bowen, 1988; Bowen and Willson, 1991; Blocker and Schonberner, 1991; Vassiliadis and Wood, 1993).

As they approach the SW phase, AGB stars become invisible at optical wavelengths and very infrared (IR) bright. During the SW stage, intense mass loss depletes the remaining hydrogen in the star's outer envelope, and terminates the AGB phase. The rapid depletion of material from the outer envelope of the star means that while AGB mass loss may last for $>10^{5} \mathrm{yrs}$, this extremely high mass-loss SW phase must have a relatively short duration (a few $\times 10^{4}$ years; Volk et al. (2000)).

In fact, heavy mass loss causes AGB stars to lose mass at such tremendous rates that they 
wither into white dwarfs rather than explode as supernovae. For a more detailed description of AGB stars we refer to Habing (1996) and Habing and Olofsson (2004).

\section{Chemistry}

The stability of the $\mathrm{CO}$ molecule in the stellar atmosphere means that the $\mathrm{C} / \mathrm{O}$ controls the chemistry around the star: whichever element is less abundant will be entirely locked into $\mathrm{CO}$ molecules, leaving the more abundant element to control dust formation. Therefore AGB stars (and their progeny, PNe) can be either carbon- $(\mathrm{C}-)$ rich or oxygen- $(\mathrm{O}-)$ rich. For O-rich AGB stars, $\mathrm{C} / \mathrm{O}$ can vary from approximately cosmic $\mathrm{C} / \mathrm{O} \approx 0.4$ ) to just less than unity. Once $\mathrm{C} / \mathrm{O}$ is greater than unity these stars become $\mathrm{C}$-rich. Other nuclear processes (e.g. the s-process) also occur in the He- and H-burning shells of AGB stars and thus other new elements are also dredged up and enrich the dust formation region.

During their ascent of the AGB, LIMS evolve chemically, starting with oxygen-rich atmospheres. Helium-burning forms ${ }^{12} \mathrm{C}$ deep inside the star. A combination of this nucleosynthesis with thermal instability and internal opacity gives rise to dredge-up events in which newly-formed $\mathrm{C}$-atoms are transferred to the surface of the star. Thus, carbon is injected into the stellar atmosphere. In some cases, carbon-to-oxygen ratio $(\mathrm{C} / \mathrm{O})$ eventually exceeds unity and transforms the star's chemistry from oxygen $(\mathrm{O})$-rich carbon(c)-rich.

It is expected that the dust formation process will follow a dust condensation sequence (Tielens, 1990; Stencel et al., 1990; Gail and Sedlmayr, 1999; Lodders and Fegley, 1999), which describes the order in which various dust species condense from a cooling gas. For O-rich stars the resulting dust species will be O-rich, e.g. magnesium-iron (Mg-Fe) silicates and oxide and calcium-aluminium $(\mathrm{Ca}-\mathrm{Al})$ compounds. In contrast, when $\mathrm{C} / \mathrm{O}>1$, the excess $\mathrm{C}$ leads to the formation of $\mathrm{C}$-rich dust species like graphite/amorphous carbon and 
carbides as well as sulfides.

There are empirical relations that suggest that (1) molecular hydrogen emission is correlated with bipolarity of PNe (Zuckerman and Gatley, 1988); and (2) bipolarity is correlated with C-richness of PNe (Zuckerman and Aller, 1986; Rola and Stasińska, 1994). Thus investigating the effect of chemistry $(\mathrm{C} / \mathrm{O})$ on the prevalence and survival of smallscale molecular structures is important.

\section{Post-AGB evolution}

Once the AGB star has exhausted its outer envelope, the mass loss virtually stops and the AGB phase ends. At this stage, the circumstellar envelope expands and drifts away from the central star, becoming optically thin after a few hundred years.

Meanwhile, the star rapidly evolves to higher temperatures at constant luminosity. As the star collapses under gravity, it heats up from $\sim 3000 \mathrm{~K}$ until it is hot enough to ionize the surrounding gas $(>30,000 \mathrm{~K})$, at which point the object becomes a planetary nebula $(\mathrm{PN})$. The short-lived post-AGB phase, as the star evolves toward to the PN phase, is also known as the proto- or pre-planetary nebula (PPN) phase. However, not all post-AGB stars will become PNe; for some post-AGB objects the expansion speed of the circumstellar shell, combined with its density will preclude a visible nebula of ionized gas ${ }^{2}$.

\subsection{Planetary Nebula: A Star's Dying Breath}

Planetary nebulae, the near-end stage for LIMS, are the short-lived, transitional states between AGB stars and white dwarfs that are pivotal to the redistribution of material to the

\footnotetext{
${ }^{2}$ Indeed, the term pre-PN was adopted to replace proto-PN to reflect the idea that not all PPNe will end up as PNe.
} 
ISM. The current model for PNe consists of ionization of the circumstellar envelope created during the AGB phase, by the now very hot remains of the central star.

While fundamental, this model cannot alone account for observations of PNe. These objects host a myriad of beautiful, yet scientifically perplexing morphologies, chemistries and appearances. As such, a standard model of the PN has been the subject of interest and debate for 30 years. Early models for the structure of PNe invoked a limb-brightened sphere dominated by ionized emission. In such models, UV radiation from the central star creates an onion-layer structure of concentric shells in which the level of ionization decreases with distance from the central star. These early models also define PNe to be either density- or ionization-bounded. In a density-bounded PN, the nebula is fully ionized and the "edge" of the nebula is where the density of ionized gas is low enough to be considered ISM. In an ionization-bounded $\mathrm{PN}$, the ionization front is still propagating into neutral gas and marks the edge of the nebula. It has become clear that most PNe are axisymmetric/bipolar and many exhibit molecular emission from within the ionized zone. Moreover, a single PN may be ionization-bounded in one plane and density-bounded in another, because it is not spherically symmetric. Simple models no longer suffice and mysteries abound (see Sahai (2009)).

Early observations of PN found higher expansion velocities, higher densities, and welldefined shells rather than the slower, less dense, and diffuse AGB envelope. Kwok et al. (1978) successfully accounted for this by introducing the Interacting Stellar Winds (ISW) model. During the post-AGB phase, the stellar wind has a lower density but higher speed than the previous AGB outflow. In the ISW model, this so-called "fast wind" sweeps up the slower moving AGB wind into a dense circumstellar shell. Advances in technology provided high resolution images for studying PNe. Unfortunately, these studies raise more 


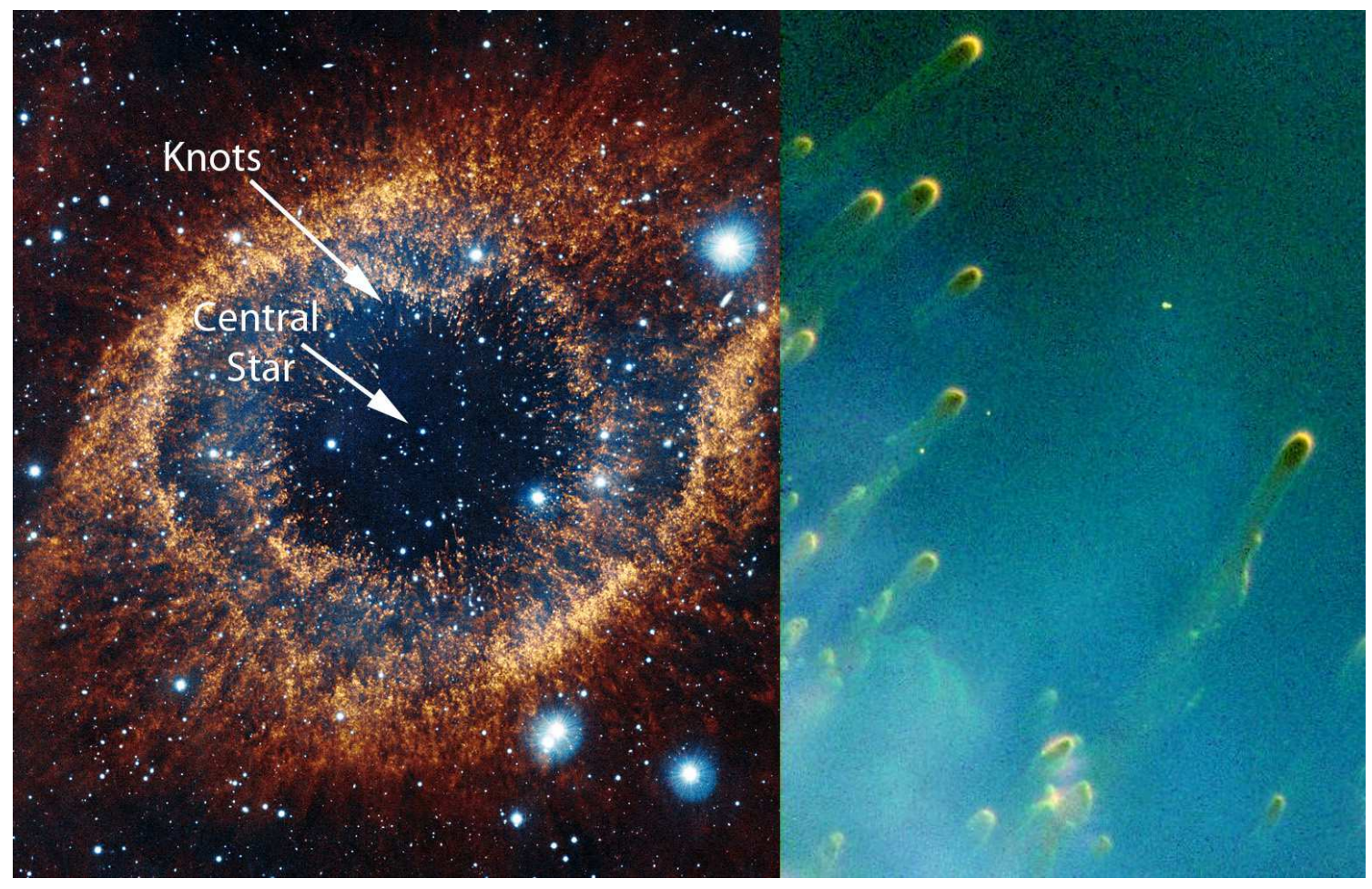

Figure 2.1: The Helix Nebula. Left: Infrared image of Helix nebula. Credit: ESO/VISTA/J. Emerson; RighT: HST image of cometary knots. Credit: NASA,C.R. O'Dell;

questions than have been answered. Simple formation models (e.g. ISW model) can no longer completely account for all the observed properties of PNe.

\subsubsection{Morphology}

Bipolarity in PNe, while common, is not well understood. Suggested models for the cause of this bipolarity include: large progenitor mass, close binaries, magnetic fields, and stellar rotation. These models are not yet definitive, nor easily testable in many cases. While AGB stars generally have spherically-symmetric mass loss (e.g., Olofsson et al. (2000), most post-AGB stars and PNe show axisymmetric circumstellar envelopes (Meixner et al., 1999; Sahai, 2004; Soker and Subag, 2005). The onset of the axisymmetry is believed to occur 
very near the end of the AGB phase (e.g., Oloffson (2004)). While some PNe are indeed spherical (Soker and Subag, 2005), these appear to be old nebulae, and it is increasingly evident that the majority of PNe are aspherical and most are bipolar (Soker and Subag, 2005).

The generalized interacting stellar wind (GISW) model has been proposed to explain the transition from a spherically symmetric AGB wind to a bipolar PPN and subsequent PN (e.g., Kwok (1982); Kahn and West (1985). In this model, a dust torus forms around the star during AGB phase, shielding equatorial regions surrounding the star from subsequent fast wind during PPN and PN phases. The resulting shape can be described as hourglass or bow-tie. The exact origin of the torus is not specified. Suggested mechanisms for the formation of a dust torus and/or bipolar PNe include: (1) binary interactions (e.g., Harpaz and Soker (1994); Mastrodemos and Morris (1998); Soker (2000); Boffin et al. (2012) (2) stellar magnetic fields (e.g., Blackman et al. (2001); Nordhaus et al. (2007), or (3) rapid star rotation (e.g., Dorfi and Hofner (1996); Dijkstra and Speck (2006a), with some overlap. For a more detailed review of competing mechanisms see Dijkstra and Speck (2006b). The current leading theory requires a binary system to drive bipolarity. A survey of known binary PNe show that in each case, the binary system creates a bipolar PNe perpendicular to the orbital plane of the companion star (Jones et al., 2012).

A survey of PNe done by Zuckerman and Gatley (1988) found that all PNe which exhibit $\mathrm{H}_{2}$ emission at $2.12 \mu \mathrm{m}$ were also found to be bipolar (Gatley's Rule). It was also found that most bipolar PNe are discovered at low galactic latitude (Zuckerman and Gatley, 1988; Kastner et al., 1996), which could signify relatively high-mass progenitor stars or higher metallicity progenitor stars. These conclusions are based solely on observations and the physics behind bipolarity and richness in $\mathrm{H}_{2}$ remains unknown. The apparent correlation 
between C-richness and bipolarity complicates the ideas further.

\subsubsection{Small-Scale Structures}

Advanced astronomical technologies led to higher resolution imaging which revealed many small-scale features inside PNe. Heterogeneities and filamentary structures in the ionized emission from PNe have been known for many years (e.g. Curtis (1918); Aller (1954); Zanstra (1955)). About $50 \mathrm{PNe}$ are presently known to have small-scale heterogeneities located inside or outside the main ionized nebulae (onçalves et al., 2001). Moreover, it is often the case that these small scale structures exhibit molecular emission from within the ionized region of the nebula where molecular material should not be able to exist (e.g. Kastner et al. (1996); Speck et al. (2002).

Probably the most famous small-scale structures in PNe are the cometary knots in the Helix Nebula, which account for all $\mathrm{H}_{2}$ emission in the nebula (e.g. Speck et al. (2002); Meixner et al. (2005); Matsuura et al. (2009)). In both the Helix and Ring nebulae, this molecular emission has been shown to emanate from dense condensations of molecular material in the form of cometary knots; named as such for the similarity in appearance to comets: a dense head with a tail pointed away from the central star. This discovery led to the revision of the simplistic, ionized bubble model for PNe. Incorporation within optically thick (i.e., opaque) knots could provide the protection required for molecular material to survive. Thus, the new paradigm for the structure of planetary nebulae involves dense knots of material. The cometary knots/molecular clumps themselves raise a myriad of interesting questions that relate to the formation and evolution of not only the knots themselves, but also the entire nebula. 
Knot formation Hypothetical mechanisms for knot formation can be divided into two basic categories: primordial (i.e., formed during AGB phase; e.g. Dyson et al. (1989)) or in situ with the onset of the PN phase (e.g. Capriotti (1971); Visniac (1994)). In each case there are several pathways to knot formation and initial density enhancements later evolve into what we see as knots.

For the primordial formation hypothesis, knots were already formed during AGB phase and sufficiently dense clumps can become self-shielded from harsh radiation and can survive through the post-AGB/pre-PN phase into the PN phase where they are sculpted by ionization and/or winds/shocks to be seen as knots (Redman et al., 2003). Density enhancements can occur in AGB circumstellar shells via several distinct mechanisms. Inhomogeneities on the stellar surface propagate into the atmosphere and into the AGB slow wind causing clumpiness. However, even a smooth outflow containing only one dust grain species will become turbulent and create density enhancements (Woitke, 2006). Meaburn et al. (1998) measured the expansion velocities of several knots in the Helix nebula and found they are consistent with AGB wind velocities rather than PN wind velocities suggesting that the outward motion of the knots is consistent with a primordial origin rather than in-situ. Another possible mechanism for density enhancements during the AGB phase is the formation of spiral shocks by interaction with a close binary companion (Edgar et al., 2008). This coincides with current hypotheses for the bipolar structure of PNe being linked to binary interactions as suggested by (De Marco, 2009). In the case of the Dumbbell nebula, binary interactions remain plausible due to the possible presence of a companion star of spectral type M5 V (De Marco et al., 2013).

For the case of in situ knot formation, density enhancements occur as a result of either the interacting stellar winds (shock front) or the effect of the ionization front. The fossil AGB 
circumstellar shell is assumed to have a more or less smooth distribution, but the knots form at the ionization or shock front due to Rayleigh-Taylor instabilities (e.g. Capriotti (1971); Visniac (1994)). An alternative in situ mechanisms suggests that a reduction in the fast wind can lead to fragmentation of a smooth nebula via depressurization. Hydrodynamical simulations from García-Segura et al. (2006) demonstrated that such a scenario gives rise to knots with comet-like tails.

Knot shaping Cometary knots come in a variety of shapes. High-resolution imaging shows a diverse range of knot structures (see Fig. 2.2 (right), and Matsuura et al. (2009)). In particular, the shapes range from comet-like to tadpole-like and the tails can be straight, narrowing, widening, or meandering from the knot-head. Mechanisms for shaping knots depend heavily on the knot-formation mechanisms. However, the shaping mechanism(s) must account for the observed knot shapes. Three shaping mechanisms have been proposed: streaming, photoevaporation, and shadowing effects. In reality, a combination of two or more may be at work.

Streaming is the hydrodynamic interaction between the wind and the "head" of the knot (e.g., Zanstra (1955); Visniac (1994); Pittard et al. (2005); Dyson et al. (2006); Pittard et al. (2009)). The differential velocity between the knot and the surrounding gas forms a bowshock in the head of the knot causing a crescent shape (Matsuura et al., 2007). The tail is a result of ram pressure; narrowing for subsonic ambient winds, and widening for supersonic ambient winds (Pittard et al., 2005). The ambient winds flow from the knot-head itself.

Photoevaporation models involve the interaction between ionizing UV radiation from the central star and the surface of the knot-head (e.g., Mellema et al. (1998); Williams 
(2003); García-Segura (2005)). The knot-head heats up and photoevaporates on the sunny side and forms the crescent tip. This model cannot account for the variations in tail shape, such as widening, narrowing and meandering of the tail. Williams (2003) predicted a jet shape based on ionization front instabilities but no observational evidence has been found to support this model yet.

Finally, the shadowing effect assumes that the knot-tail does not see the ionizing radiation from the central star because it is in the shadow of the knot-head (e.g., Soker (1998)). This model can only produce straight tails and thus cannot be solely responsible for knot shaping.

Five PNe were studied in detail by O'Dell et al. (2002), who found that these small-scale structures seem to be common, if not ubiquitous. Furthermore, they found that there is a progression in the appearance of these globules that correlates with the age (evolutionary status) of the nebula, starting with globules closest to the central star having dark tangential structures, which are not aligned with the central star and are located near the main ionization front. These structures do not have bright edges. In a slightly older PN the structures are still dark (no bright edges) but show well-defined globules and have some indications of tails (e.g. the Ring Nebula; Fig. 2.2.2). Finally, the globules become "knots" with very well defined with bright, photo-ionized edges facing the central star and tails radially oriented away from the central star (e.g. the Helix Nebula; Fig. 2.2).

Meixner et al. (2005) compared $\mathrm{H}_{2}$ emission in the Helix nebula, seen with Hubble Space Telescope (HST) NICMOS observations, to its ionized gas emission and demonstrated that the morphology of the knots changes with distance from the central star, and revealed the intricate structure of the knots.

Therefore, the globule-shaping mechanisms must be able to account not only for the range of shapes of knot-heads and tails, but also for the evolution in the observed shapes, 


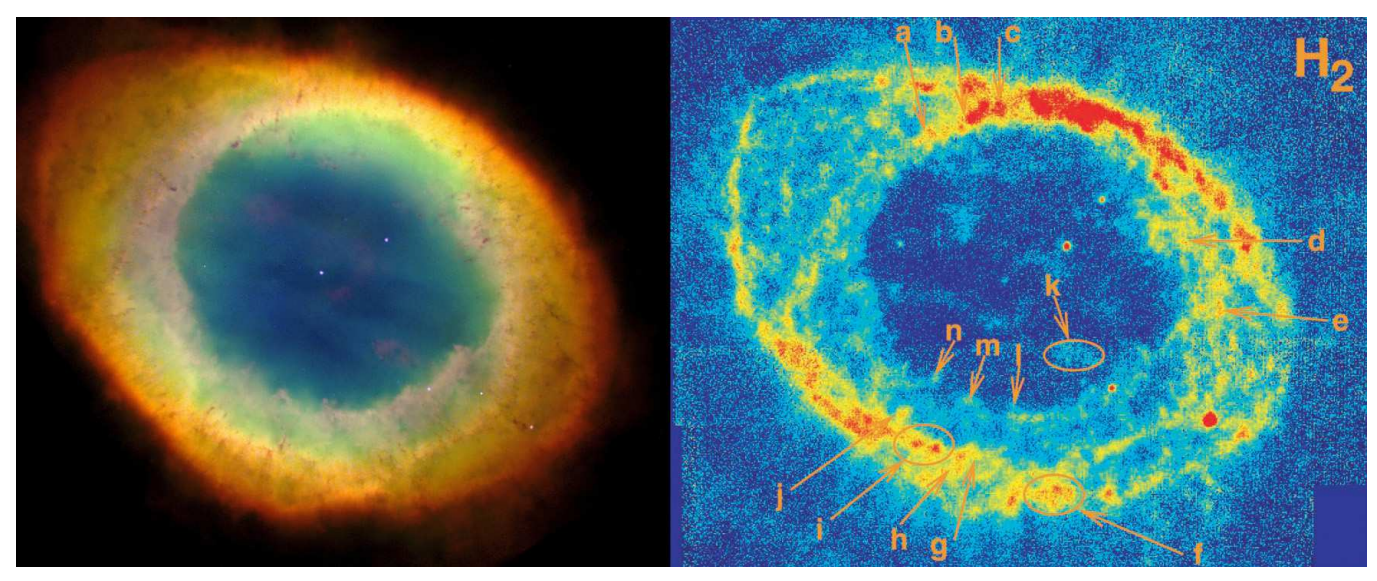

Figure 2.2: The Ring Nebula. Left: HST Visible. Image credit: The Hubble Heritage Team (AURA/STScI/NASA); Right: Ground based $\mathrm{H}_{2}$ image $(2.12 \mu \mathrm{m})$. Image credit: Speck et al. (2003).

and the variations in shapes both from PN to PN and within a single PN.

\subsubsection{Molecular Hydrogen}

Molecular hydrogen $\left(\mathrm{H}_{2}\right)$ is the most common molecule in the Universe and therefore often used as a tracer for molecular material in PNe. For example, the $2.12 \mu \mathrm{m} \mathrm{v}=1-0 \mathrm{~S}(1)$ ro-vibrational line has been used to image $\mathrm{H}_{2}$ in several PNe (e.g. Speck et al. (2002, 2003); Meixner et al. (2005); Matsuura et al. (2009); Marquez-Lugo et al. (2013)). $\mathrm{H}_{2}$ not only provides a reliable means of imaging molecular gas in $\mathrm{PNe}$, but understanding its formation and excitation mechanisms provide clues to the formation and evolution of the knots, in turn building a more complete picture of PNe dynamics.

Despite having no dipole moment, $\mathrm{H}_{2}$ can be detected due to transitions of the electronic ground state as a result of changes in ro-vibrational or pure rotational quantum states (see 2.1 , where $v=$ vibrational quantum number and $J=$ rotational quantum number). The resultant emission due to changing energy levels exhibits energies typically found in the 
near- to mid-IR. ${ }^{3}$

$$
E=\left(v+\frac{1}{2}\right) h v_{0}+h c \bar{B} J(J+1)
$$

In PNe, this emission occurs due to either shock or fluorescent excitation. In shock or collisional excitation, the $\mathrm{H}_{2}$ is given kinetic energy from collisions and subsequent emission results in the line intensities following a thermal population distribution. Fluorescent excitation arises from UV photons from the central star, which excite the $\mathrm{H}_{2}$, resulting in a cascade down to lower energies. Each of these mechanisms has corresponding line intensity ratios which can be used to determine insights into excitation methods.

However, excitation mechanisms for $\mathrm{H}_{2}$ in $\mathrm{PNe}$ are not well constrained. When $\mathrm{H}_{2}$ was first imaged in PNe, the observed brightnesses were found to be too high to be explained by fluorescent excitation (i.e., emission from photon-dominated or photo-dissociation regions; PDRs) and thus $\mathrm{H}_{2}$ in PNe was assumed to be shock excited (Zuckerman and Gatley, 1988).

The original PDR models were developed to understand interstellar molecular clouds, and were not appropriate to the circumstellar nebulae around rapidly evolving stars. These early PDR models only included far-UV photons and failed to include the soft X-ray emission inherent from very hot $(>100,000 \mathrm{~K})$ white dwarf central stars of PNe. Further, the models did not account for the changing temperature of central star or the expansion of the nebula. The interstellar models assume a smooth slab of material rather than a clumpy medium. Natta \& Hollenbach (1998; NH98) successfully included all of these factors (evolving central star, expanding gas, x-ray photons, clumping) into their PDR model for PNe and showed that the intensity of the $\mathrm{H}_{2}$ emission was consistent with fluorescent excitation in

\footnotetext{
${ }^{3} \mathrm{~A}$ shorthand is used to describe specific transitions, for example, $v=1 \rightarrow 0 \mathrm{~S}(1)$ represents a change in vibrational quantum number from $\mathrm{v}=1$ to $\mathrm{v}=0$ and $\mathrm{S}(1)$ represents a rotational change of $\Delta \mathrm{J}=-2 \mathrm{with}$ a final $\mathrm{J}=1$. The example transition has an emission at $2.122 \mu \mathrm{m}$.
} 
PDRs. The NH98 model has been successfully applied to three PNe, NGC2346 (Vivini et al., 1999), the Helix nebula citepSpeck2002, Speck2003) and the Ring Nebula (Speck et al. 2003; see Fig. 1. of Speck et al. 2003). Consequently, $\mathrm{H}_{2}$ excitation in PDRs became favored (e.g. Latter, Dayal, Beiging et al. (2000); Hora et al. (1999); Latter, Dayal, Bieging et al. (2000); Speck et al. (2002, 2003); Huggins et al. (2002)).

In the case of the Helix nebula, the spectrum of individual knots was obtained in the range of 2-5 $\mu \mathrm{m}$ which is populated by the 1-0 and 2-1 ro-vibrational lines (Matsuura et al. 2007). The observed line ratios could not be explained by the fluorescent excitation due to the stellar UV flux. As a result, these observations favor either purely shock-excited $\mathrm{H}_{2}$ emission or perhaps a combination of both fluorescent and shock excitation.

$\mathbf{H}_{2}$ Formation In the ISM, the formation of $\mathrm{H}_{2}$ is typically attributed to formation on dust grains due to insufficient densities to form in free space (Hollenbach and Salpeter, 1971). However, in higher densities and the presence of ionized hydrogen, as found in $\mathrm{PNe}$, other formation mechanisms can become significant. According to Aleman and Gruenwald (2004) and Aleman and Gruenwald (2011) the most important reactions for the formation of $\mathrm{H}_{2}$ in $\mathrm{PNe}$ are:

$$
\begin{gathered}
2 \mathrm{H}+\text { grain } \rightarrow \mathrm{H}_{2}+\text { grain } \\
H+H^{-} \rightarrow H_{2}+e^{-} \\
H_{2}^{+}+H \rightarrow H_{2}+H^{+} \quad 4
\end{gathered}
$$




$$
H_{3}^{+}+e^{-} \rightarrow H_{2}+H^{5}
$$

In previous studies, it has been found that the observed $\mathrm{H}_{2}$ emission in the Helix nebula arises completely from knots (Speck et al. 2002, Meixner et al. 2005, Matsuura et al. 2009). Whether this is common among all PNe which show excited $\mathrm{H}_{2}$ is not yet determined, however the presence of knots and the formation $\mathrm{H}_{2}$ are believed to be linked. As such, the formation of $\mathrm{H}_{2}$ molecules may depend on the formation mechanisms for the knots themselves. If the $\mathrm{H}_{2}$ pre-exists the $\mathrm{PN}$, then the knots act to shield molecules from dissociation by stellar UV photons, allowing for continued survival. If $\mathrm{H}_{2}$ is formed in-situ in $\mathrm{PNe}$, then the formation process must be promoted by the inherent high densities and low temperatures in the knots.

Cometary Knots and Molecular Hydrogen Due to their relative small size, the discovery of knot-bearing PNe is limited by distance and current telescope capabilities. Only a handful of nebulae are sufficiently close such that the knots can be resolved and even less have been extensively probed for $\mathrm{H}_{2}$ emission. However, despite the small sample size, determining a link between cometary knots and $\mathrm{H}_{2}$ is being pursued. Table 2.1 summarizes a selection of knot-bearing nebulae.

The following two chapters (Chapters $3 \& 4$ ) look at two paradigm-challenging case studies, The Dumbbell Nebula and NGC 2392, in order to determine the extent of $\mathrm{H}_{2}$ emission from cometary knots or otherwise in these two nebulae.

\footnotetext{
${ }^{4} \mathrm{H}_{2}^{+}$can be effectively formed by the reactions: $\mathrm{H}^{+}+\mathrm{H}^{-} \rightarrow \mathrm{H}_{2}^{+}+e^{-}$and $\mathrm{H}+\mathrm{H}^{+} \rightarrow \mathrm{H}_{2}^{+}+h v$

${ }^{5}$ Formation mechanism is only important in knots (Aleman et al. 2001)
} 
Table 2.1: List of PNe with observable knots and whether they exhibit any $\mathrm{H}_{2}$ emission

\begin{tabular}{lcccl}
\hline $\begin{array}{l}\text { Nebula } \\
\text { Name }\end{array}$ & $\begin{array}{c}\text { Distance } \\
(\mathrm{pc})\end{array}$ & $\begin{array}{c}\mathrm{H}_{2} \\
\text { detected? }\end{array}$ & $\begin{array}{c}\text { Globules } \\
\text { detected? }\end{array}$ & Comments \\
\hline IC 4406 & 600 & unknown & yes & \\
NGC 2392 & 1300 & no & yes & this work (Chapter 4) \\
NGC 5189 & 550 & unknown & yes & \\
Mz-3 & 2500 & unknown & yes & \\
\hline NGC 2346 & 700 & yes & yes & \\
Helix & 215 & yes & yes & \\
Ring & 600 & yes & yes & \\
Dumbbell & 405 & yes & yes & this work (Chapter 3) \\
\hline
\end{tabular}




\section{Chapter 3}

\section{The Dumbbell Nebula}

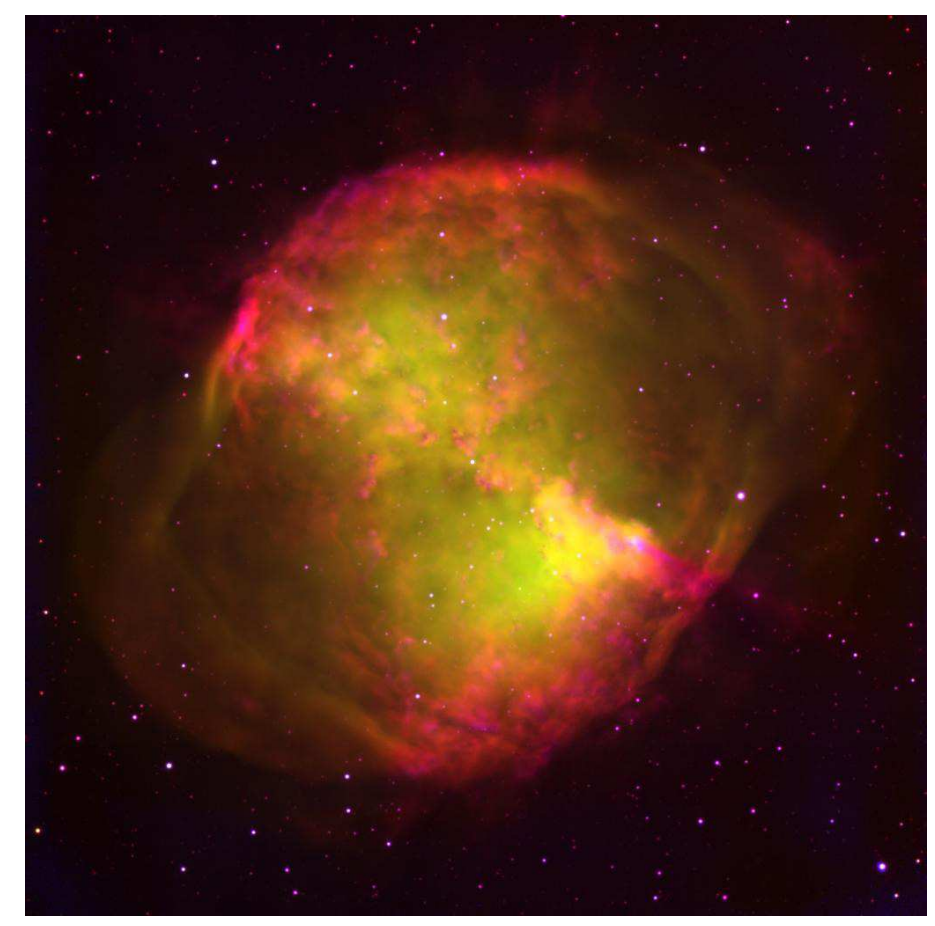

Figure 3.1: Color composite image of the Dumbbell nebula. Credit: George Jacoby, WIYN telescope 


\subsection{Characteristics}

The Dumbbell nebula (M27) is one of the closer planetary nebula to Earth with a distance of $405 \mathrm{pc}$ and is the first planetary nebula to be discovered by Charles Messier in 1764 (Benedict et al., 2009). Extending an area $8^{\prime} \times 5.6^{\prime}$, the Dumbbell is an excellent target for which to observe small-scale structures. Visually, the Dumbbell is a bipolar nebula with bright twin triangular lobes extending away from the central star from NE to SW (see Figure 3). The majority of $\mathrm{H}_{2}$ emission and cometary knots exist in this region. A second, more circular, more diffuse region extends around the whole nebula and is slightly elongated 90 degrees to the bright region (NW to SE). There is little to no cometary knots or $\mathrm{H}_{2}$ emission in this region.

The morphology of the Dumbbell is clearly bipolar, however it is difficult to determine the exact shape of a 3 dimensional object from its 2 dimensional projection. Kwok et al. (2008) attempt to explain the shape of the Dumbbell with a pair of triple biconic structures based upon 2.12um $\mathrm{H}_{2}$ images. Kwok et al. (2008) goes on to predict that the Ring nebula follows the same shape however its projection oriented "down the barrel" of such a biconic structure, however more recent structure models of the Ring nebula suggest a torus (O'Dell et al., 2013) which would put it in the same morphological class as the Helix nebula and NGC 2346. 


\subsection{Observations and data reduction}

\subsubsection{Near-IR (Subaru)}

\section{$2.12 \mu \mathrm{m}$ image}

Near-IR images of the Dumbbell nebula were taken by MOIRCS on the Subaru telescope at Mauna Kea, Hawaii, USA on 2007 June 25 (UT). Sky subtraction was done by telescope nodding off source. Dithering was also used to reduce hot pixels. The total exposure was $100 \mathrm{~s} \times 8$ on source. Throughout the observation, the air mass was around 1.09 and seeing was $0.49^{\prime \prime}$ in the near-IR (FWHM) measured by stars within the field. MOIRCS's strength is in its large field of view $\left(4^{\prime} \times 7^{\prime}\right)$ enabled by two HAWAII-2 arrays, each $2048 \times 2048$ pixels and a pixel scale of $0.117^{\prime \prime}$. A narrow band filter was used with a wavelength range of $2.116 \pm 0.021 \mu \mathrm{m}$, measured by FWHM. At this wavelength, the $\mathrm{H}_{2} v=1 \rightarrow 0 \mathrm{~S}(1)$ line is dominant and continuum emission from the nebula is likely negligible (Matsuura et al. 2007).

We use IRAF and the IRAF-based software QMCS which has been developed by one of the authors (I.T.) for the data reduction. We first adopted the correction of the flat field, the sky subtraction, and the distortion correction, and a mosaic image was created from eight target frames by registering field stars and by adopting masks on bad pixels. In the final image, there are low level artifacts in the form of multiple circular rings on the image taken with one of the arrays. This pattern is caused by interference of the filter. In the $\mathrm{CH} 1$ image (the south part of the image), this pattern was reduced using No. 2, 2009 H2 KNOTS IN NGC 72931069 defringe?moircs.pro, which is a part of SIMPLE Imaging and Mosaicking Pipeline (Wang, 2010). The median of the rms noise distribution is taken as the sensitivity. 
We use Two Micron All Sky Survey (2MASS) magnitudes of three stars in CH1, and we assume that their Ks magnitudes are identical in the $\mathrm{H} 2$ filter.

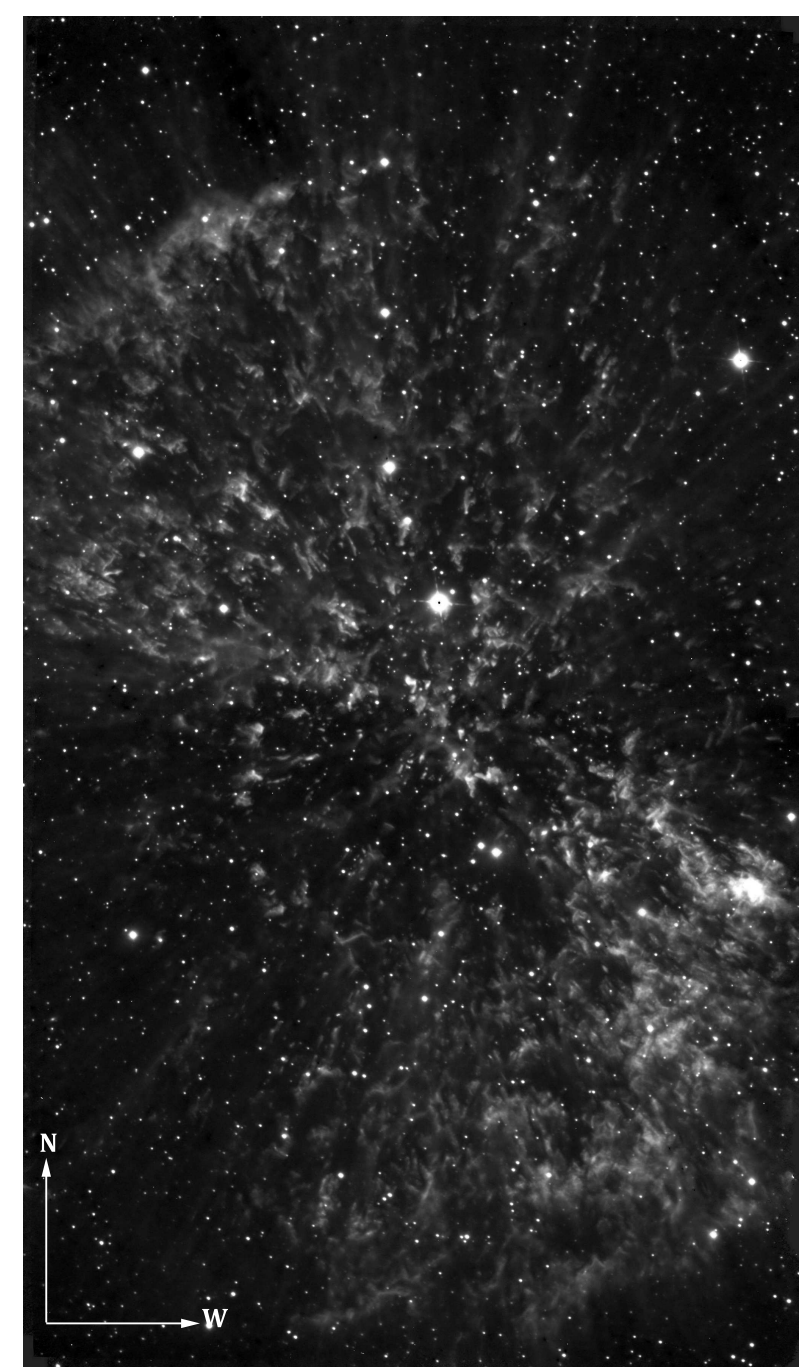

Figure 3.2: $\mathrm{H}_{2}$ image of Dumbbell Nebula taken by MOIRCS on Subaru

\section{Multi object spectroscopy}

Multi-object, long slit spectroscopy was obtained by MOIRCS onboard the Subaru telescope at Mauna Kea, Hawaii, USA on 2007 August 25 (UT). Exposures were completed in a 

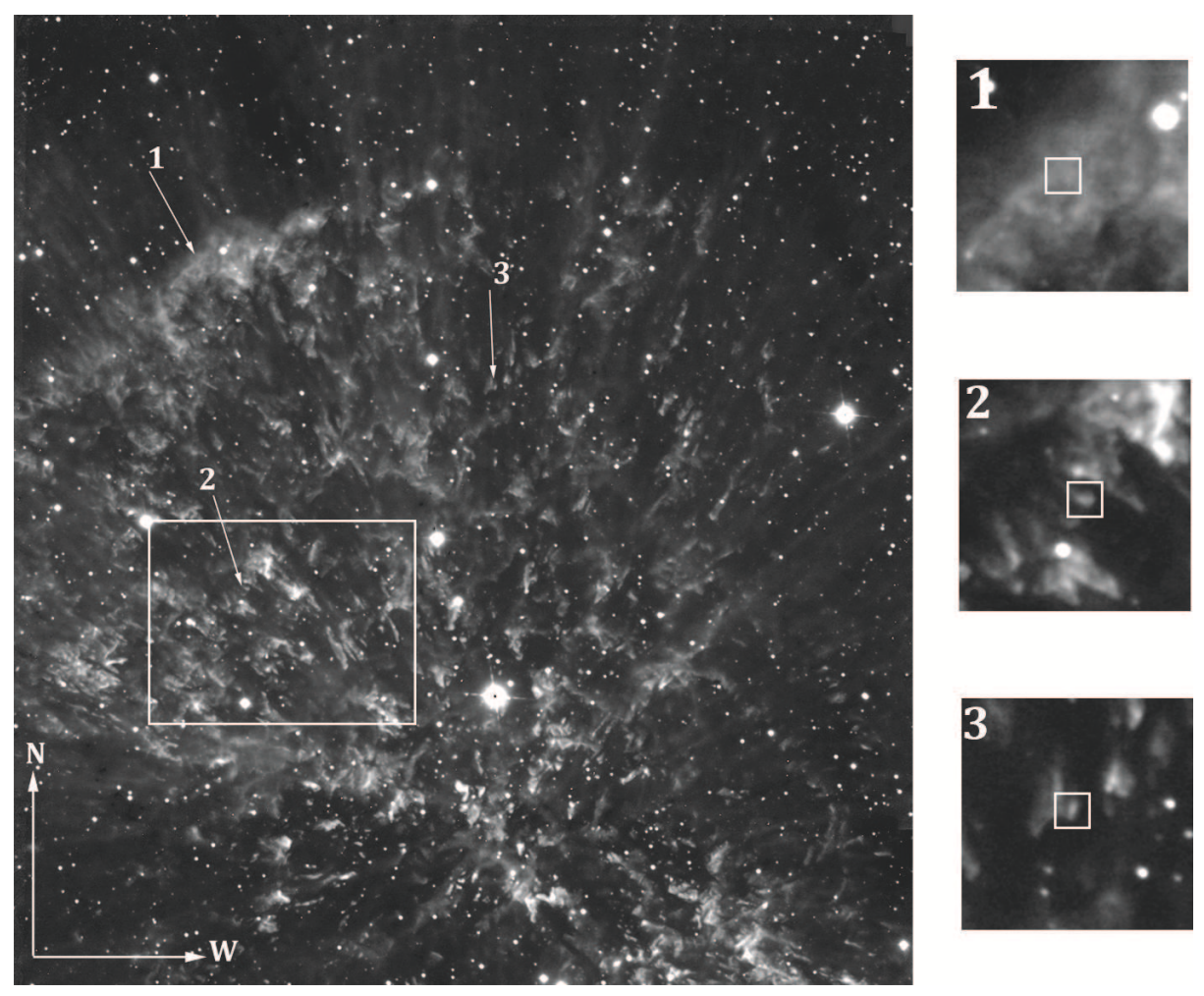

Figure 3.3: Map of slit locations used on MOIRCS; The box represents the zoomed region in Figure 3.9.

repeated three step sequence of: 300 s on source $\rightarrow 300$ s offset $3.5^{\prime \prime}$ on source $\rightarrow 300$ s sky off source. Total integration time on source was $12 \times 300$ s, total integration time of sky was $6 \times 300$ s. Throughout the observation, the air mass varied from 1.01-1.06 and seeing was $0.55^{\prime \prime}-0.65^{\prime \prime}(\mathrm{FWHM})$ measured by stars within the field. Only the CH2 $2048 \times 2048$ pixel HAWAII-2 array was used in the spectroscopic observations. The broad band filter Ks was used in conjunction with the R1300 grism. The Ks filter has a central wavelength of $2.15 \mu \mathrm{m}$ with a width of $0.31 \mu \mathrm{m}$. Slit locations can be seen in Fig. 3.3. 


\section{Spectral data reduction}

Data reduction for spectroscopy was completed manually (ie: without pre-compiled data reduction software) using IRAF. Along with data obtained of the target, wavelength calibration frames were obtained of a Th-Ar (Thorium-Argon) lamp which has a known spectrum for comparison.

\subsubsection{Visible (WIYN)}

The Dumbbell was imaged in three narrow bands $([\mathrm{O} I]=630.0 \mathrm{~nm},[\mathrm{O} \mathrm{III}]=500.7 \mathrm{~nm}, \&$ $\mathrm{H} \alpha=656.3 \mathrm{~nm}$ ) using the WIYN MiniMosaic Imager by George Jacoby who has graciously allowed their use in this study. The WIYN Dumbbell images all have a field of view of $8.6^{\prime} \times 8.6^{\prime}$

\subsection{Analysis}

\subsubsection{Image Analysis}

The four narrow band images of the Dumbbell nebula presented in this paper include the $2.122 \mu \mathrm{m} \mathrm{S}(1)$ transition of $\mathrm{H}_{2}$, the $630.0 \mathrm{~nm}[\mathrm{O} \mathrm{I}]$ image, the $656.3 \mathrm{~nm} \mathrm{H} \alpha$ image, and the 500.7nm [O III] image (see Figs. 3.2, 3.4(a), 3.4(b), \& 3.4(c)). As one would expect in a $\mathrm{PN}$, the emission from [O III] is very bright and universally dispersed throughout the nebula and brightest at the core. Conversely. in $\mathrm{H}_{2}, \mathrm{H} \alpha$, and [O I] images, this same region corresponds with the brightest emission in the nebula. In general, dark "clouds" in [O III] image correspond to bright regions of molecular and atomic gas emission. 

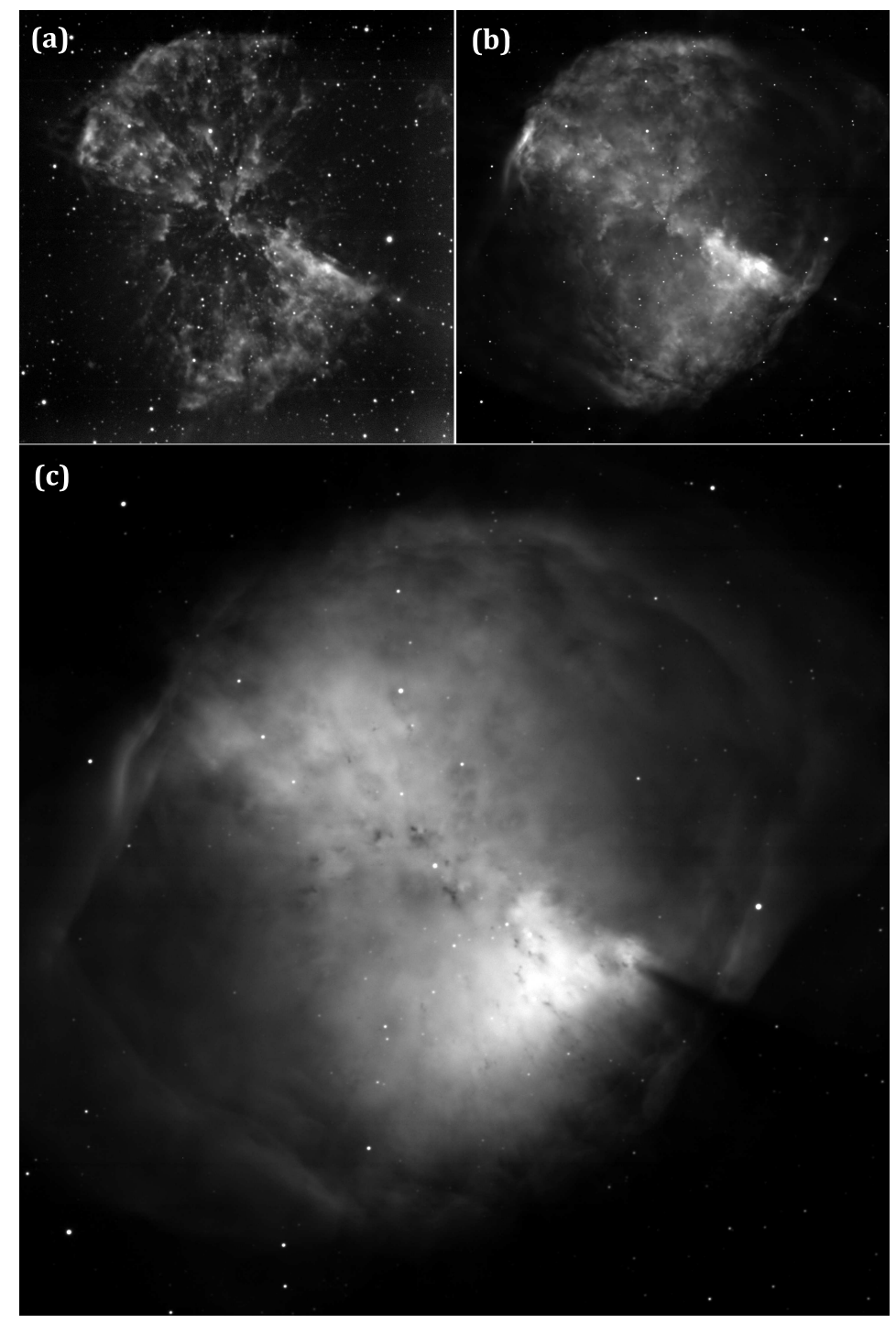

Figure 3.4: (a) [O I] image of Dumbbell Nebula taken by WIYN; (b) $\mathrm{H} \alpha$ image of the Dumbbell taken by WIYN; (c) [O III] image of the Dumbbell taken by WIYN.

Knots can be seen in the $\mathrm{H}_{2}$ image similar to those found in previous studies of the Helix (Matsuura at al. 2009) and Ring (Speck et al. 2003) nebulae. Knots seen in visible Hubble images of the Dumbbell match those seen in the $\mathrm{H}_{2}$ image. Taking a random sample of knots (20 members), the average "width" of a knot as seen in $\mathrm{H}_{2}$ (measured from widest point of the head) is roughly $0.8^{\prime \prime}$. Taking the distance of the Dumbbell to be $405 \mathrm{pc}$ (Benedict 
2009), this corresponds to an actual diameter of $344 \mathrm{AU}$.

$\mathrm{H}_{2}$ emission from the Dumbbell nebula can be associated with three different types of structures (or regions). First, as mentioned, there is emission from cometary knots previously identified by O'Dell (2002). Second, the majority of $\mathrm{H}_{2}$ emission stems from amorphous clumpy structures with no definite head or tail. Lastly, $\mathrm{H}_{2}$ emission can be seen and spectroscopically verified from diffuse regions between structures as well as in the form of "crepuscular-like" rays on the edge of the nebula. These rays are most notably seen in the NE (upper left) of Figure 3.2. Comparing the $\mathrm{H}_{2}$ emission and $\mathrm{H} \alpha$ images (see Fig. 3.5), one can make correlations between atomic and molecular hydrogen. In general, the $\mathrm{H}_{2}$ emission traces areas of atomic emission with regions of $\mathrm{H}_{2}$ being more localized rather than throughout as is the case with atomic hydrogen (i.e. $\mathrm{H}_{2}$ is clumpier). One area of note is on the edge of the nebula where molecular emission dominates and there is little seen in $\mathrm{H} \alpha$; this is most apparent in the NE region of the nebula (arrow of figure 3.5). This may indicate the leading edge of the ionization or shock front. It is also noteworthy that the molecular hydrogen emission becomes much less sculpted in this region with no apparent globules, rather, the emission looks diffuse and unstructured. Spectroscopic analysis of this region shows a similar spatial distribution (see section 3.3.2). Comparing $\mathrm{H}_{2}$ and [O I] emission show much similarity as well, with most of the emission arising from clumpy, localized regions.

The Dumbbell nebula has a galactic latitude of $-3.7^{\circ}$, this proximity to the galactic plane unfortunately makes for a dense star population in the field of view. In past observations at these wavelengths, the resolution was not high enough to adequately distinguish between flux from the field stars and flux from the nebula, thus falsely elevating surface brightness measurements from molecular hydrogen. Since the Subaru image of the Dumbbell boasts 


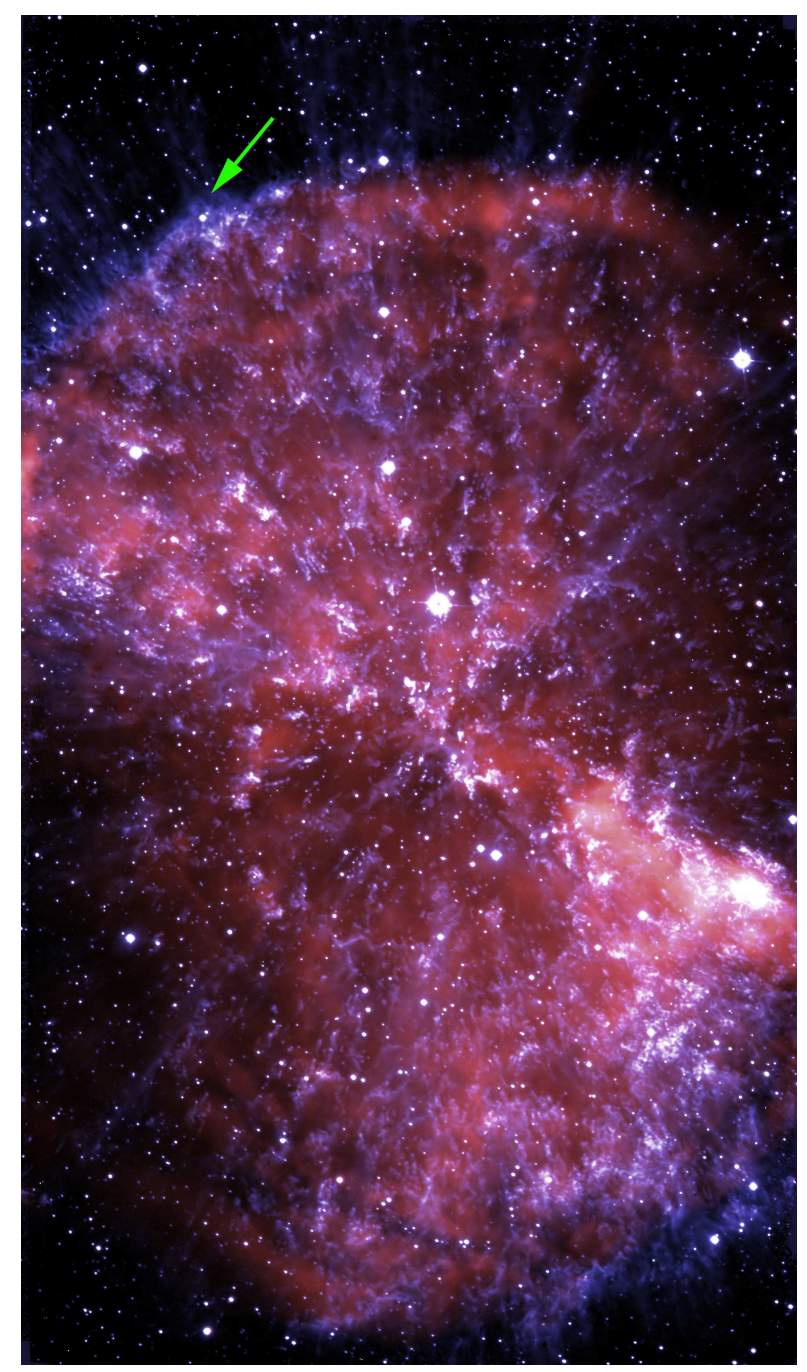

Figure 3.5: Superposition of the Subaru $\mathrm{H}_{2}$ image (blue) and WIYN $\mathrm{H} \alpha$ image (red). Arrow indicates the region where $\mathrm{H}_{2}$ dominates at the edge of the visible nebula.

very high resolution, an analysis could be completed to determine the significance of the contribution of stellar light to the overall surface brightness. Roughly 3500 stars were selected and removed from the image by means of a simple "slicing off" of each star peak to a value determined by the sky average around the star. While this method of star removal is not as ideal as a Gaussian PSF subtraction for each star, it does provide an upper bound on the 
star-less flux of the $\mathrm{H}_{2}$ emission from the nebula. Average surface brightness was measured for just the "bow-tie" portion of the nebula both with and without stars for comparison. The average surface brightness with the stars is $6.1 \times 10^{-5} \mathrm{ergs} \mathrm{cm}^{-2} \mathrm{~s}^{-1} \mathrm{sr}^{-1}$ while without the stars is $5.2 \times 10^{-5} \mathrm{ergs} \mathrm{cm}^{-2} \mathrm{~s}^{-1} \mathrm{sr}^{-1}$; a $15 \%$ decrease in average surface brightness after removing the stars in field.

\subsubsection{Spectral Analysis}

We used a slit sizes with a lengths of $14 \operatorname{arcsec}$ or 9.3 arcsec and widths of 0.58 arcsec. Using the slits corresponding to regions shown in Fig. 3.3, specific regions of the nebula were selected in which the slit passed through a knot or diffuse region. Two slits adequately covered a knot which we could partition around and extract their spectrum. A third region near the edge of the nebula showed bright diffuse emission in $\mathrm{H}_{2}$ which was subsequently chosen for spectral analysis as well. The spectra for each region can be found in Figs. 3.6-3.8 and the locations of the slits are shown in Fig. 3.3. Note that the spectra have not been flux calibrated thus the vertical axis has arbitrary units.

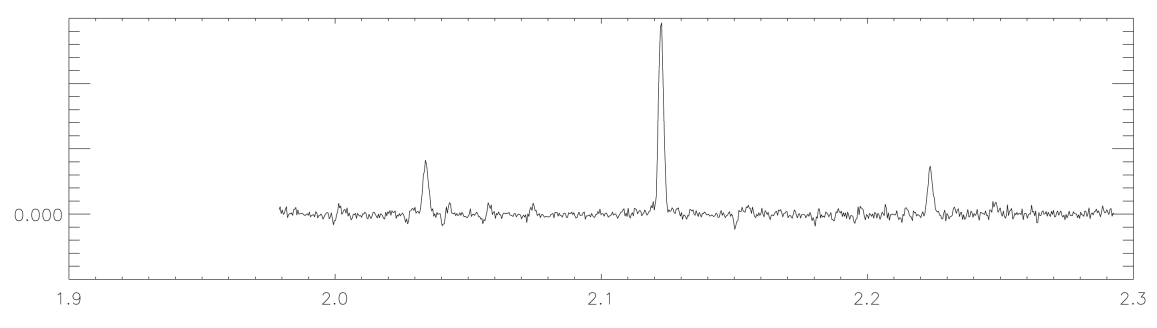

Figure 3.6: Spectrum 1 (diffuse), $x$-axis is wavelength measured in $\mu \mathrm{m}, y$-axis is flux (not calibrated)

In each spectrum, three prominent molecular hydrogen lines are observed allowing for an analysis of line ratios. The most prominent line is the $2.122 \mu \mathrm{m} \mathrm{H}_{2} v=1 \rightarrow 0 \mathrm{~S}(1)$ line, this 


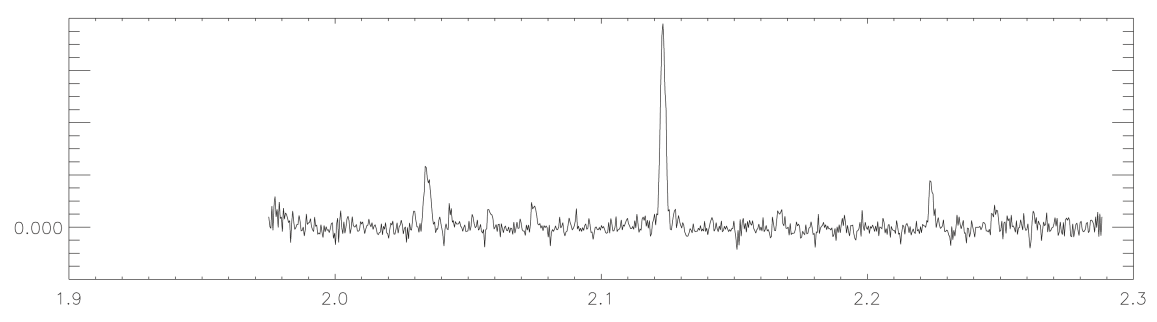

Figure 3.7: Spectrum 2 (knot), $x$-axis is wavelength measured in $\mu \mathrm{m}, y$-axis is flux (not calibrated)

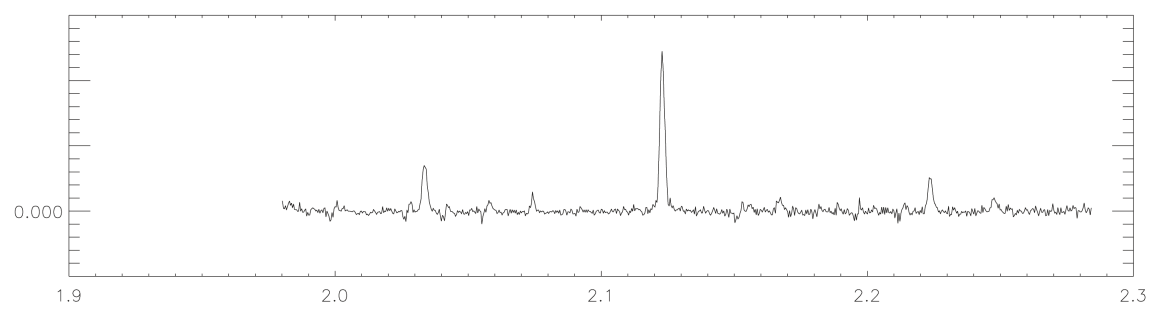

Figure 3.8: Spectrum 3 (knot), $x$-axis is wavelength measured in $\mu \mathrm{m}, y$-axis is flux (not calibrated)

is the line corresponding to the $\mathrm{H}_{2}$ image. The $2.0338 \mu \mathrm{m} \mathrm{H}_{2} \nu=1 \rightarrow 0 \mathrm{~S}(2)$ and $2.2235 \mu \mathrm{m}$ $\mathrm{H}_{2} v=1 \rightarrow 0 \mathrm{~S}(0)$ lines are also observed. A fourth $\mathrm{H}_{2}$ line is also seen in spectra with higher S/N corresponding to the $2.2477 \mu \mathrm{m} \mathrm{H}_{2} v=2 \rightarrow 1 \mathrm{~S}(1)$ line. For ease of reading, these lines will be referred to as 1-0 S(1), 1-0 S(2), 1-0 S(0), and 2-1 S(1) respectively. Another notable spectral line, corresponding to the $2.165 \mu \mathrm{m} \mathrm{Br} \gamma$ atomic hydrogen transition, can be seen in some, but not all spectra. Most notably, $\operatorname{Br} \gamma$ detection is absent in the diffuse region represented by spectrum 1 . This agrees with the image comparison between $\mathrm{H} \alpha$ emission and $\mathrm{H}_{2}$ emission which when stacked, show marked reduction in $\mathrm{H} \alpha$ flux where the diffuse $\mathrm{H}_{2}$ can be seen. This of course assumes that $\mathrm{Br} \gamma$ emission correlates with bright $\mathrm{H} \alpha$ regions.

The line ratios 1-0 S(0)/1-0 S(1) and 2-1 S(1)/1-0 S(1) were measured to determine the 
excitation temperature of $\mathrm{H}_{2}$. Error analysis in line ratios was based upon the average noise level in a flat region of the spectrum between 2.09-2.10 $\mu \mathrm{m}$. Line ratios can be found in Table 3.1. Measured line ratios in Spectrum 1 and 2 for the 2-1 S(1)/1-0 S(1) lines are low $(\sim 0.06)$ compared to predicted values from Natta \& Hollenbach (1998) of $\sim 0.1$ for a PN at this stage of its life $\left(\mathrm{T}_{*}>100,000 \mathrm{~K}\right)$.

\subsection{Discussion}

\subsubsection{Comparison to other PNe}

\section{The Helix Nebula}

We compared the $\mathrm{H}_{2}$ image of the Dumbbell with that of the Helix nebula (from Matsuura et al. 2009). In figure 3.9 we compare of a group of knots from the Helix nebula with a group of knots in the Dumbbell (the resolution of the Helix image was decreased to in order to make both Helix and Dumbbell images as if these objects were equidistant from Earth). Knots in the Helix nebula have the highest number density in two "rings" defined by O'Dell et al. (2004) and Matsuura et al. (2009) as the inner and outer rings. The knot region from the Helix is from the inner ring. The corresponding region in the Dumbbell nebula is highlighted in Figure 3.3. In each nebulae, the knots show a tendency to cluster in groups ranging from 2 to $5+$ members with tails pointed radially away from the central star. The sizes of the knots, once distance is accounted for, are remarkably similar for both nebulae. A random sampling of knot head widths were measured in each nebulae. The Dumbbell knots are roughly $0.8^{\prime \prime}$; at 405pc (Benedict 2009) this makes the diameter of a knot around $344 \mathrm{AU}$. In the original (non-degraded) image, the Helix knots are $\sim 1.5^{\prime \prime}$ across 
at a distance of $216 \mathrm{pc}$, (Benedict 2009) corresponding to $344 \mathrm{AU}$. The full extent of this similarity has yet to be studied but may be attributed to similar knot formation mechanisms. However, while the $\mathrm{H}_{2}$ emission in the Helix nebula is consistent with solely emanating from knots (Matsuura et al. 2009), this is not the case in the Dumbbell. A large portion of the emission in the Dumbbell appears to come from shapeless clumps and diffuse regions and knots are generally less sculpted in the Dumbbell as compared to the Helix.

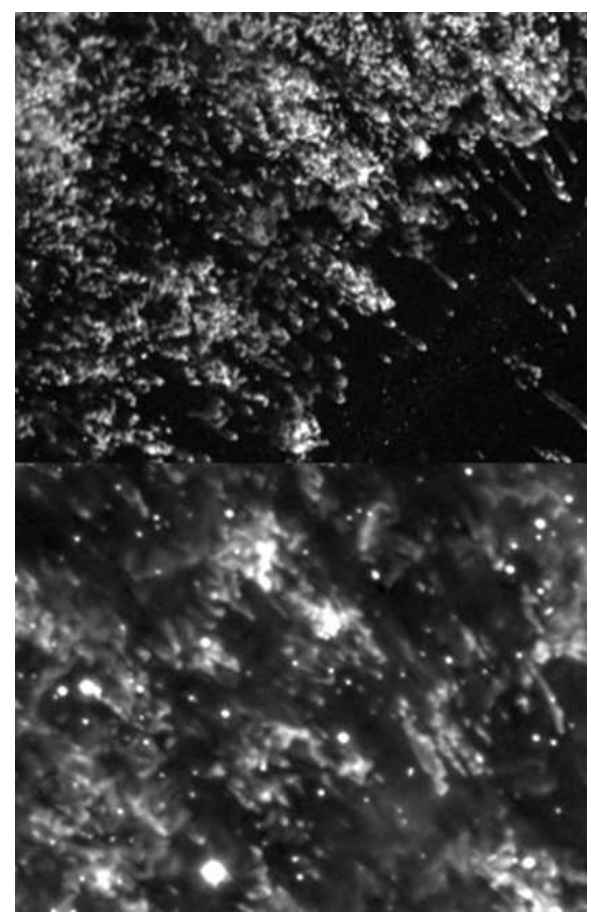

Figure 3.9: Top: Middle region of the Helix nebula in $\mathrm{H}_{2}$ (Matsuura 2009). Bottom: Region of Dumbbell nebula indicated in Figure 3.3.

Unlike the Helix nebula, which clearly exhibits a relation between knot density and sculpting with distance from the central star (CS; Matsuura et al. (2009)), the Dumbbell image shows no such relation. Instead, there is no general trend in density. In this case, intrinsic shape and orientation to our line of sight plays a large role. It is currently assumed that knots in the Helix populate a dense toroidal ring oriented such that we see along (or 
near) its primary axis (O’Dell et al. 2004). This enables measurements based on distance from the CS possible. It is difficult to know the exact orientation of the Dumbbell nebula with our line of sight. As such attempting to determine relations based on distance from the CS prove futile. Developing three-dimensional hypothetical models of planetary nebulae could help determine properties of the physical structure of the nebula in the future.

\section{The Ring Nebula}

When comparing surface brightness of the Dumbbell to that of either the Ring or Helix nebula, we must keep in consideration the differences between orientation and line of sight. Both the Ring and Helix are "ring-like" PNe, as such, most of the flux from $\mathrm{H}_{2}$ comes from a bright ring surrounding the central star. In the Ring nebula, Speck et al. (2003) determined the surface brightness of $\mathrm{H}_{2}$ of only the ring, ignoring the dark space in the

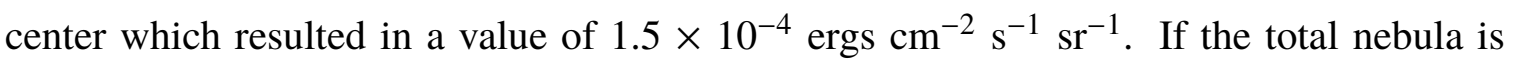
averaged including the central dark space (eg., Kastner et al. 1994) the average surface brightness drops to $7 \times 10^{-5} \mathrm{ergs}^{-2} \mathrm{~s}^{-1} \mathrm{sr}^{-1}$. The average surface brightness for the Dumbbell nebula is closer to the latter (for the Ring nebula). One might expect this if the shape of the dumbbell is simply a result of orientation, our line of sight would include both the inner dark space as well as the brighter "ring". Further analysis of the average surface brightness was performed on a smaller and brighter region of the Dumbbell located roughly $1.5 \operatorname{arcmin} \mathrm{SW}$ of the CS. The average surface brightness of this region is $1.2 \times 10^{-4} \mathrm{ergs}$ $\mathrm{cm}^{-2} \mathrm{~s}^{-1} \mathrm{sr}^{-1}$, more closely resembling that of the ring region of the Ring nebula. 


\subsubsection{Knot and $\mathrm{H}_{2}$ Formation}

In section 2.2, we discussed competing mechanisms for $\mathrm{H}_{2}$ formation in $\mathrm{PNe}$, however studies of $\mathrm{H}_{2}$ emission in the Ring and Helix nebulae (Speck et al. 2002; Speck er al. 2003; Matsuura et al. 2009) had proved unable to distinguish between proposed mechanisms. It has been shown by Aleman \& Gruenwald (2004) and Aleman et al. (2011) that $\mathrm{H}_{2}$ can be formed and survive in ionized regions within PNe, this gives a means for continued $\mathrm{H}_{2}$ formation after onset of PNe phase. In the models put forth in the aforementioned papers, $\mathrm{H}_{2}$ formation was most prominent in the transition zone between regions of high ionization and neutral gas. The outer region which exhibits un-clumped $\mathrm{H}_{2}$ emission (Spectrum 3 as seen in Figure 3.3) could represent such a region. Comparing the $\mathrm{H}_{2}$ and [O I] images reveals that this region has high surface brightness in both images. This also agrees with Aleman et al. (2011) which predicts $\mathrm{H}_{2}$ emission from bright [N II], [O I], and [S II] regions.

Unfortunately, emission models for $\mathrm{H}_{2}$ in cometary knots (Aleman et al. 2011) based on the Helix nebula rely on estimates of knot distance from the CS. In the case of the Dumbbell, estimates of knot distances from the CSPN and the luminosity and temperature of the CSPN are difficult without knowing the true shape and orientation of the nebula from our perspective. This remains an issue when studying PNe in general, however headway is being made: e.g. O'Dell et al. (2013) has successfully developed a 3D model of the Ring nebula which fits observations. Similar work on other nebulae (including the Dumbbell) could help further our understanding of small scale structures in PNe. 


\subsubsection{Excitation Temperatures}

In order to determine possible excitation mechanisms of the $\mathrm{H}_{2}$, we looked at the ratio of line intensities between the $v=1-0 S(0), S(1), S(2)$ and $v=2-1 S(1)$ lines. A summary of these ratios is found in table 3.1. Each line was fit by a Gaussian and the integrated flux of that fit used in the calculations. Using the methods put forth in Smith et al. (1997), hereafter SDL), the excitation temperatures $\left(T_{e x}\right)$ ratios were determined for two regions within the nebula. The regions of the nebula were selected based on morphology and location, specifically a knot and diffuse region in the outer nebula. Rotational excitation temperatures can be determined utilizing the $v=1-0$ ratios and should, for the same region, be roughly the same. Vibrational excitation temperatures can be determined by ratio of the $v=2-1 \mathrm{~S}(1)$ and $v=1-0$ S(1) lines. A comparison between the vibrational and rotational excitation temperatures can be used to determine possible excitation methods behind the $\mathrm{H}_{2}$ emission; assuming local thermal equilibrium. The SDL model used for determining excitation temperatures is shown below in equation 3.1. The energies for each transition $(E)$, the wavelengths $(\lambda)$, and statistical weights $(g)$ were taken from Dabrowski (1984) while the Einstein coefficients (A) were taken from Turner et al. (1977). In our case, the intensity of each line, $I$, is the integrated spectral feature.

$$
T_{e x}=\frac{E_{1}-E_{2}}{\ln \left(\frac{I_{1} A_{2} \lambda_{1} g_{2}}{I_{2} A_{1} \lambda_{2} g_{1}}\right)}
$$

In Spectrum 1, which is a diffuse region on the edge of the nebula, analysis of excitation temperatures in this region shows an average rotational excitation temperature of $1140 \pm 60$ $\mathrm{K}$ and a vibrational excitation temperature of $2020 \pm 90 \mathrm{~K}$. Spectrum 2, which is taken at the head of a knot within the nebula, we find an average rotational excitation temperature of 
$1480 \pm 170 \mathrm{~K}$ and a vibrational excitation temperature of $2180 \pm 230 \mathrm{~K}$. Long slit spectroscopy was used to obtain the spectra, therefore detailed analysis of possible variations across the individual knot was impossible. Multiple spectra obtained of a knot in high spatial resolution (such as integral field spectroscopy) would be ideal.

Table 3.1: Line ratios and excitation temperatures of $\mathrm{H}_{2}$ Near-IR lines.

\begin{tabular}{l|ccc|ccc}
\hline & \multicolumn{3}{|c|}{ Spectrum 1, Diffuse } & \multicolumn{3}{c}{ Spectrum 2, Knot } \\
Lines & Ratio & $T_{e x}(\mathrm{~K})$ & Err $(\mathrm{K})$ & Ratio & $T_{e x}(\mathrm{~K})$ & Err $(\mathrm{K})$ \\
\hline $1-0 \mathrm{~S}(0) / 1-0 \mathrm{~S}(2)$ & 0.862 & 1130 & 70 & 0.684 & 1480 & 200 \\
$1-0 \mathrm{~S}(2) / 1-0 \mathrm{~S}(1)$ & 0.322 & 1350 & 140 & 0.332 & 1450 & 270 \\
$1-0 \mathrm{~S}(0) / 1-0 \mathrm{~S}(1)$ & 0.278 & 925 & 90 & 0.227 & 1510 & 370 \\
$2-1 \mathrm{~S}(1) / 1-0 \mathrm{~S}(1)$ & 0.0851 & 2020 & 90 & 0.104 & 2180 & 230 \\
\hline
\end{tabular}

Our results show that $\mathrm{T}_{e x, v i b}$ is consistently higher than $\mathrm{T}_{e x, r o t}$ which, according to SDL, could be indicative of shock theory where gas is heated and allowed to cool unhindered or as a result of a multiple shock strengths (e.g. supersonic turbulance or curved shock fronts). Additionally, both rotational and vibrational excitation temperatures in the knot are higher than the outer diffuse region. This is likely due to the knots being closer to the center of the nebulae where both shocks and UV flux are more intense.

\subsection{Conclusions}

Molecular hydrogen emission in the Dumbbell arises from 3 distinct morphological "structures" within the nebula: Cometary knots, as seen in the Helix and Ring nebulae (among others); Amorphous clumps throughout the nebula; and diffuse (unstructured) regions, most pronounced near the edge of the nebula. When comparing the $\mathrm{H}_{2}$ image to $\mathrm{H} \alpha$, a clear boundary becomes apparent in the edges of the nebula where $\mathrm{H}_{2}$ emission becomes unstructured and the $\mathrm{H} \alpha$ emission drops off. This could indicate a shock or ionization frontal 
boundary which would imply that knots are sculpted by these fronts moving through a more homogeneous distribution of gas as seen in this region.

Regarding the entire nebular $\mathrm{H}_{2}$ emission, surface brightness measurements resemble those found in previous studies of the Ring nebula (Speck et al. 2003, Kastner et al. 1994). Average surface brightness in the Dumbbell was measured at a value lower than that found in the Ring nebula by Speck et al. (2003) yet similar to that found in the Ring nebula by Kastner et al. (1994) which included the central region of the Ring in the average. Excitation temperatures of $\mathrm{H}_{2}$ in the Dumbbell are consistent with values seen in other $\mathrm{PNe}$ (1000-2000 K) however the difference between rotational and vibrational temperatures in the Dumbbell is contrary to findings in the Helix which would signify a larger contribution of fluorescent excitation opposed to purely shock excited.

In comparison to other nebulae (The Helix and Ring nebulae), the Dumbbell's differences stand out more than the similarities. Neither the Ring nor the Helix exhibit diffuse $\mathrm{H}_{2}$ emission and in general the Dumbbell's knots are less sculpted. The differences between the morphological structure (toroidal vs. biconic) of these nebulae may play a role in these findings and in any case must be accounted for in any knot-bearing PN model. 


\section{Chapter 4}

\section{NGC 2392}

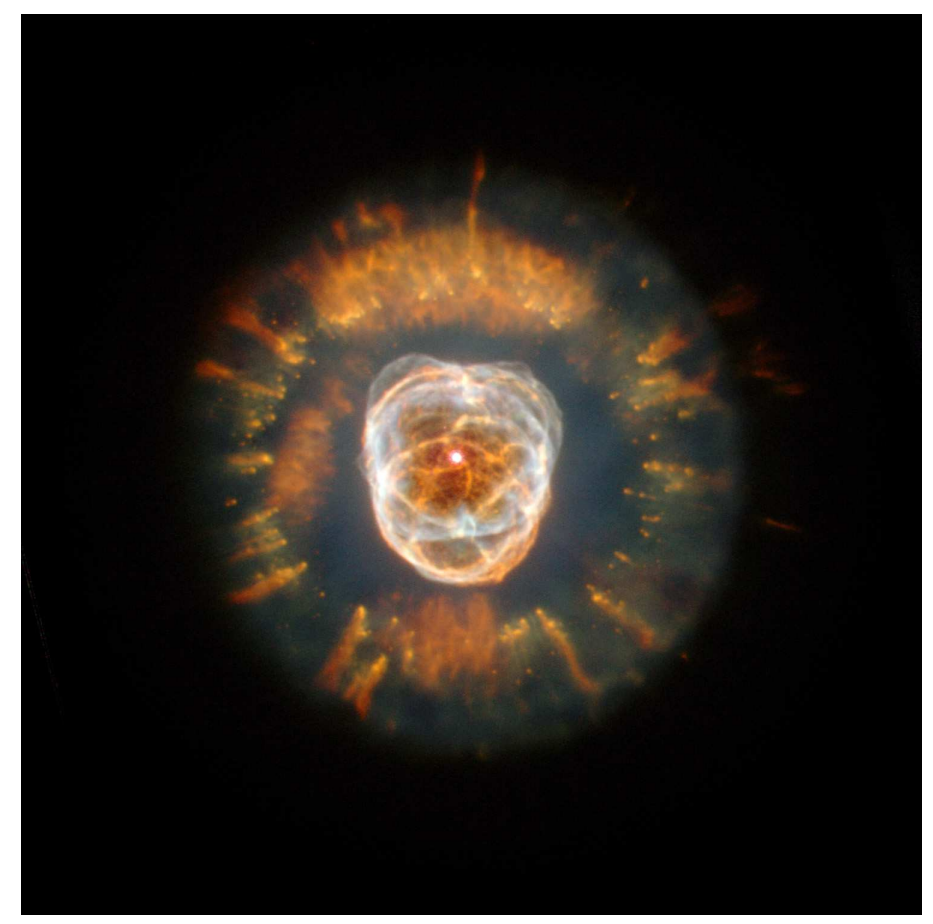

Figure 4.1: HST image of NGC 2392. Credit: Andrew Fruchter (STScI, NASA) 


\subsection{Characteristics}

NGC 2392 is a well-studied, highly recognizable planetary nebula (PN) bearing the resemblance of a face surrounded by a nearly circular ring of fur which has prompted names such as the 'Eskimo' or 'Clown-face' nebula.' High resolution visible imaging of this nebula shows that the outer ring of "fur" comprises clumps and filamentary structures reminiscent of those seen in the Helix Nebula and dubbed "cometary knots". Although the knots in the Helix are known to contain molecular hydrogen $\left(\mathrm{H}_{2}\right)$, near-infrared studies of NCG 2392 have not detected $\mathrm{H}_{2}$ gas in this nebula. The study of the clumps and filamentary structures in NGC 2392 is important to our understanding of such structures in other planetary nebulae and potentially on the survival of molecular material ejected into the interstellar medium. In this chapter we revisit both spectroscopic and imaging observations of NGC 2392 in order to address the bigger picture of PN evolution.

\subsection{Previous Observations}

Based on visible, narrow band images from HST, NGC 2392 consists of a bright region near the core of the nebula making up the 'face' which contains filament structures extending between 7 " -9 " from the central star and a region surrounding the core making up the 'fur' which contain cometary knots. The outer knot region begins between $14^{\prime \prime}-18^{\prime \prime}$ from the central star extending out to 24" (O’Dell et al., 2002). Both of these features appear to be embedded within a diffuse, nearly circular halo which extends $23^{\prime \prime}$ from the central star (see

\footnotetext{
${ }^{1}$ It is commonly known as the Eskimo nebula and was originally named for its resemblance to a person wearing a parka hood, however, the term 'Eskimo' is often considered a derogatory term for aboriginal people in the northern most areas of the Earth. With this in mind, we will only refer to the nebula as NGC 2392 and urge the astronomy community to consider a new common name. Our suggestion is the 'Lion's Head' nebula.
} 
4). However, determining the actual size of features and the dynamical age depend heavily on its distance, which is not well constrained. O'Dell et al. (2002) proposed a distance of $880 \mathrm{pc}$ and dynamical age of $\sim 1000 \mathrm{yr}$ based on a tangential expansion rate of 5 mas $\mathrm{yr}^{-1}$ (Hajian et al., 1995). More recently, using proper motions, García-Díaz et al. (2015) determined a distance of $1300 \mathrm{pc}$.

Radial velocity measurements based on doppler shifts were performed in detail by García-Díaz et al. (2012) which determined the velocity of the central star of the planetary nebula (CSPN) to be $70 \mathrm{~km} \mathrm{~s}^{-1}$ and is adopted as the system velocity. Taking into account the system velocity, the inner region is expanding at a rate of $120 \mathrm{~km} \mathrm{~s}^{-1}$ while the outer halo is expanding much more slowly at $16 \mathrm{~km} \mathrm{~s}^{-1}$. If we assume the outer halo is remnant of the AGB (Asymptotic Giant Branch) wind, then this is in agreement with the interacting stellar winds model for PNe (Kwok, 1982) which postulates a fast, post-AGB wind interacting with a slower AGB wind. The knots have a radial velocity near system velocity which suggests they form a ring perpendicular to our line of sight surrounding the central star. Based on these velocity measures and adopting a distance of $1300 \mathrm{pc}$, the dynamical age of the outer halo is 9,200 years, consistent with the end of the AGB phase. Both O'Dell et al. (2002) and García-Díaz et al. (2012) estimate that the true shape of the inner bubble is a prolate spheroid with the major axis oriented toward Earth and a ratio of 1.8 to the minor axis. With an average angular size of $16^{\prime \prime}$, the width of the spheroid would measure approximately $3.1 \times 10^{12} \mathrm{~km}$ assuming a distance of $1300 \mathrm{pc}$. The major axis would therefore measure $5.6 \times 10^{12} \mathrm{~km}$. Based on its very rapid expansion rate of $120 \mathrm{~km} \mathrm{~s}^{-1}$, the dynamical age is only 740 years assuming no acceleration. These dynamical ages suggest that NGC 2392 is a young PN primarily consisting of two expanding masses of gas: the slow moving late AGB wind making up the halo and a high speed post-AGB wind making up the inner bubble. 
The progenitor mass of the star is an estimated $1.7 \mathrm{M}_{\odot}$ based on nebular abundances (Pottasch et al., 2008). The effective temperature measurements $\left(T_{\text {eff }}\right)$ of the CSPN is model dependent and varies according to whether it calculated from the relative abundances of different ions or from the occurrence of species with higher orders of ionization. Observations of Fe IV/Fe V and $\mathrm{He} \mathrm{I} / \mathrm{He}$ II ionization balance yield a $T_{\text {eff }}$ of $40,000 \mathrm{~K}$ and $45,000 \mathrm{~K}$ respectively (Pauldrach et al., 2004; Kudritzki et al., 1997). A similar $T_{\text {eff }}=40,000 \mathrm{~K}$ based on the blackbody continuum was estimated by Pottasch et al. (1978). Models predict a CSPN $T_{\text {eff }}$ of $<40,000 \mathrm{~K}$ for a PN of this age and progenitor mass (Natta and Hollenbach, 1998) which is consistent with the aforementioned temperatures. However, the ionized helium Zanstra temperature $\left(\mathrm{T}_{z, \mathrm{He} \text { II }}=78,000 \mathrm{~K}\right)$ and the presence of high energy ions, e.g. $[\mathrm{Ne} \mathrm{V}]$ 13426, suggest a harsher UV radiation field that is not consistent with a star at $40,000 \mathrm{~K}$. This has led to higher estimates of the CPSN effective temperature $\left(T_{\mathrm{eff}}=80,000 \mathrm{~K}\right)$ or the possibility of a faint, very hot companion star with $T_{\text {eff }}=250,000 \mathrm{~K}$ (Danehkar et al., 2012). Alternatively, the high energy ion abundances could be attributed to shocks, thereby removing the need for higher estimations of the CSPN temperature or "hot companion".

Previous studies have been done on NGC 2392 in order to determine the extent of $\mathrm{H}_{2}$ emission in the nebula (Latter, Dayal, Beiging et al., 2000; Kastner et al., 1996). These studies were unable to detect the presence of any $\mathrm{H}_{2}$. However, nearly two decades on, we now have access to more advanced technologies and thus this paper revisits observations of $\mathrm{H}_{2}$ in NGC 2392. 


\subsection{Observations}

The images were taken over a series of four nights (December 3,5 2014 and January 1,2 2015) with the WIYN High Resolution Infrared Camera (WHIRC) on the WIYN 3.5 meter telescope. Typical seeing during the four nights was between $0.5^{\prime \prime}$ and $0.8^{\prime \prime}$ with average seeing around $0.65^{\prime \prime}$ at $2 \mu \mathrm{m}$. Weather conditions were generally clear with occasional thin clouds, however elevated relative humidity levels $(70 \%-90 \%)$ were present each night. Three narrow band images were obtained around $2.1 \mu \mathrm{m}$ using the $\mathrm{H}_{2} \mathrm{~S}(1), \mathrm{Br} \gamma$, and $\mathrm{Br} \gamma$ $4500 \mathrm{~km} / \mathrm{s}$ offset (henceforth Brg45) filters. Each image is a sum of several 120 second, flat fielded and sky subtracted frames with total integration times of: 202 minutes in the $\mathrm{H}_{2} \mathrm{~S}(1)$, 94 minutes in $\operatorname{Br} \gamma$, and 168 minutes in Brg45 filters. These observations are considerably longer than the previous 10 minute exposure presented by Latter, Dayal, Beiging et al. (2000). The standard star, P309-U with Ks magnitude of 11.45 (Persson et al., 1998), was

also measured in the $\mathrm{Ks}, \mathrm{H}_{2} \mathrm{~S}(1)$, and $\mathrm{Br} \gamma$ filters for flux calibration. The Brg45 image was flux calibrated based on the flux calibrated central star in the $\operatorname{Br} \gamma$ image. The resulting images as well as select HST images can be seen in Figure 4.2.

\subsection{Image and Spectral Analysis}

In order to determine the extent of $\mathrm{H}_{2}$ emission in the knots of NGC 2392 or otherwise, the three narrow band images obtained represent the source $\left(\mathrm{H}_{2} \mathrm{~S}(1)\right.$ filter), continuum $(\mathrm{Brg} 45$ filter), and comparative (Br $\gamma$ filter) flux. The $2.12 \mu$ m image was obtained through the $\mathrm{H}_{2}$ $\mathrm{S}(1)$ filter centered at $2.117 \mu \mathrm{m}$ with a bandwidth of $0.0216 \mu \mathrm{m}$. The possible contributors to the flux in this band are the $\mathrm{H}_{2} \mathrm{v}=1-0 \mathrm{~S}(1)$ line at $2.122 \mu \mathrm{m}$, which is our target emission line, and the He I line at $2.112 \mu \mathrm{m}$. The Brg45 image, with a central wavelength of $2.188 \mu \mathrm{m}$ with 


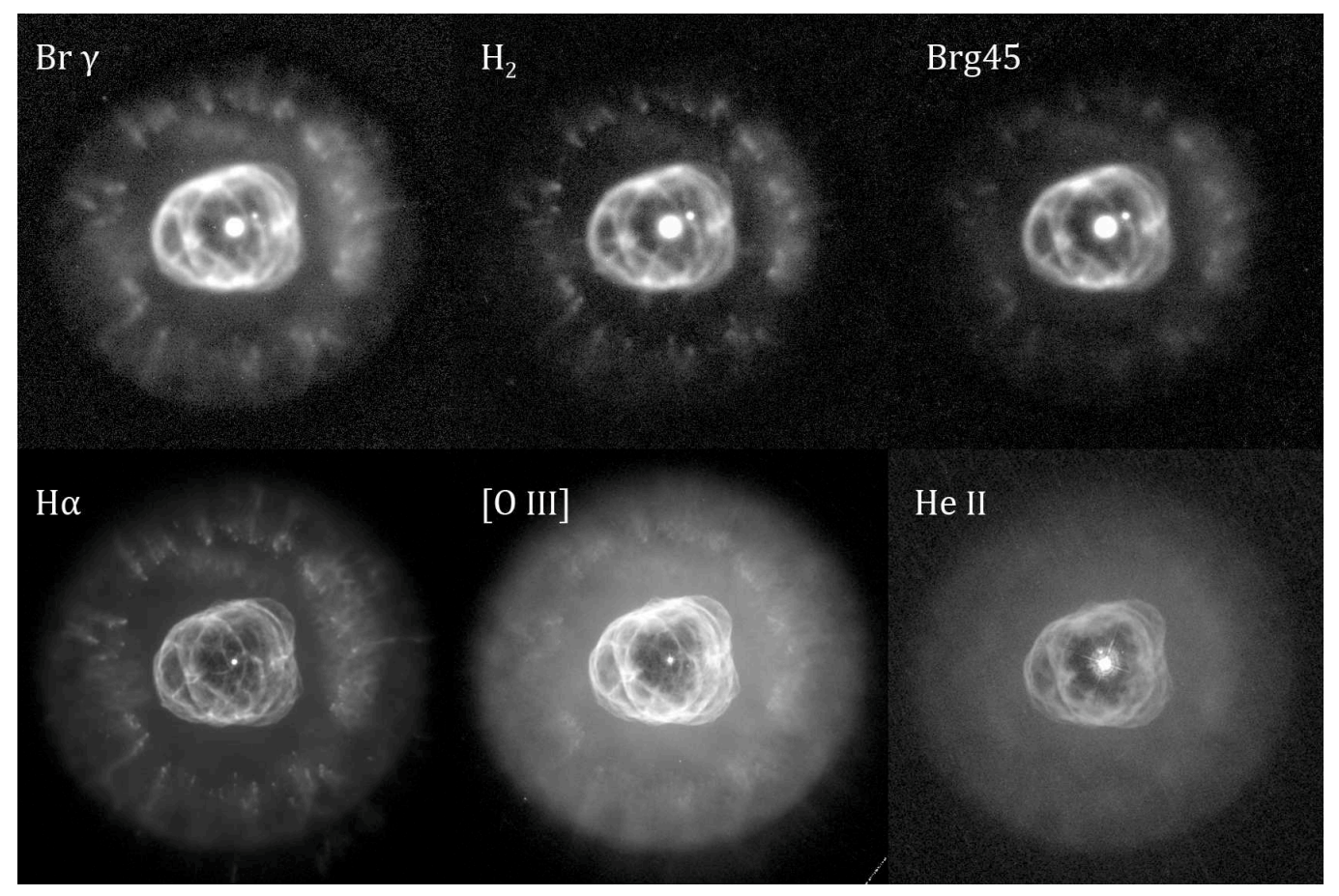

Figure 4.2: Narrow band images of NGC 2392 around $2 \mu \mathrm{m}$ taken at WIYN (top) and visible narrow band HST images (bottom). 


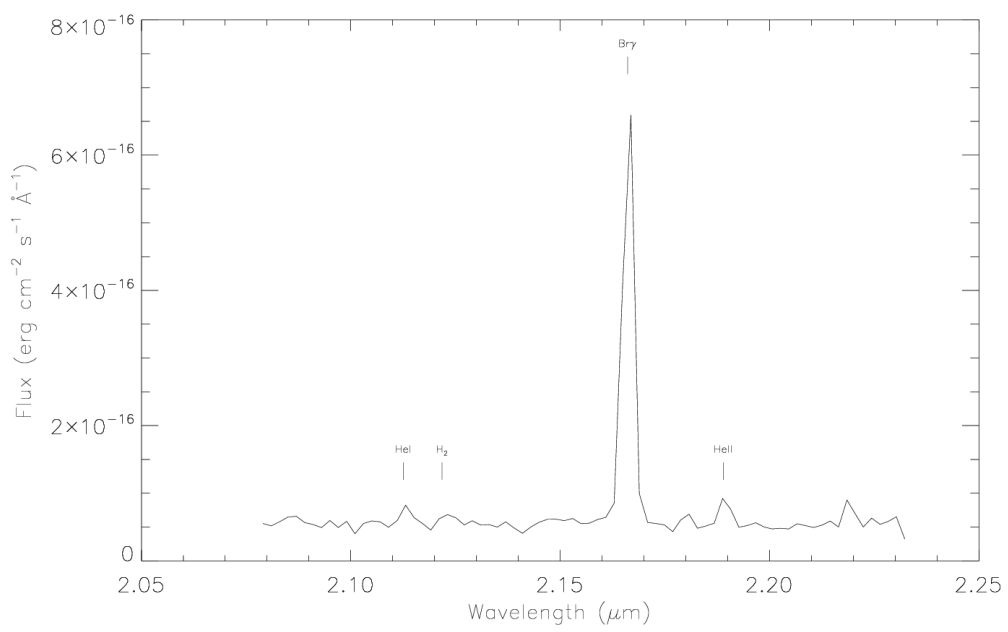

Figure 4.3: K-band spectrum obtained by H99 of the inner region of NGC 2392

a bandwidth of $0.0237 \mu \mathrm{m}$, may contain $\mathrm{Kr}$ III $(2.199 \mu \mathrm{m})$ and He II $(2.189 \mu \mathrm{m})$ emission, which have both been seen in other PNe (Likkel et al., 2006). K-band spectroscopy of the brightest portion of the nebula directly east of the CSPN ((Hora et al., 1999), henceforth H99; reproduced in Figure 4.3), suggest weak contributions from both He I and He II in the central region at ratios of 0.049 and 0.072 to $\mathrm{Br} \gamma$, respectively. There is no significant spectroscopic evidence of $\mathrm{H}_{2}$ in the $\mathrm{H} 99$ spectral region. Comparing the flux ratio of the $2.12 \mu \mathrm{m}$ image to the $\mathrm{Brg} 45$ image, we find the ratios much higher than the line ratios measured from $\mathrm{H} 99$. We can then assume the flux of the central region is a combination of $\mathrm{He} \mathrm{II}+$ continuum in the case of $\mathrm{Brg} 45$, and $\mathrm{He} \mathrm{I}+$ continuum in the $2.12 \mu \mathrm{m}$ image. This is in agreement with flux calibrated comparison of the two images $(2.12 \mu \mathrm{m}$ and Brg45) which show nearly identical flux for the central regions (Figure 4.4).

We will henceforth focus on the knot region of the $2.12 \mu \mathrm{m}$ image, which shows marked increase in flux over the Brg45 image. In the northern portion of the nebula, where the increased flux is most evident, one knot was singled out (indicated with arrow in Figure 4.4) for detailed flux comparison. The flux of a $2.485 \operatorname{arcsec}^{2}$ area encompassing the knot 


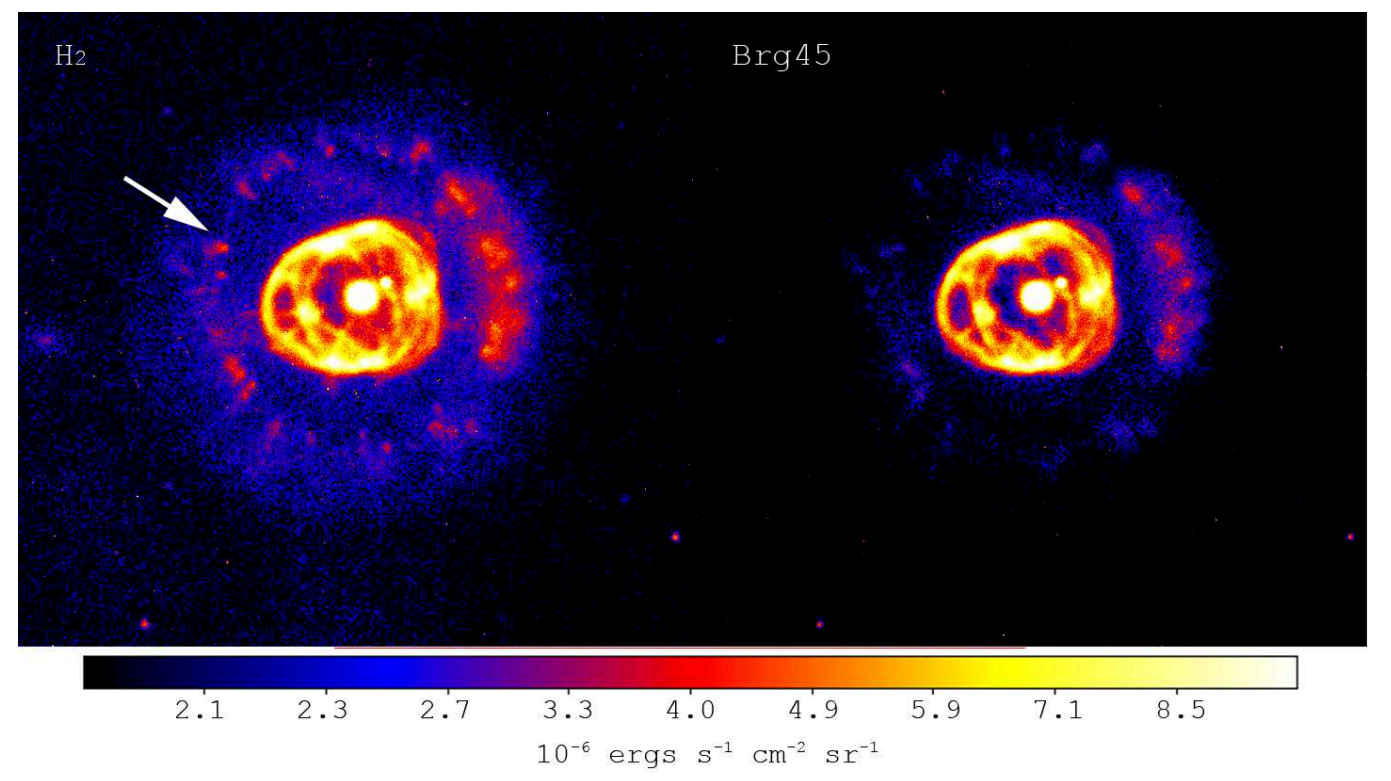

Figure 4.4: Flux calibrated images of $\mathrm{H}_{2}$ (left) and $\mathrm{Brg} 45$ (right). Scale bar is in units of surface brightness $\left(10^{-6} \mathrm{ergs} \mathrm{cm}^{-2} \mathrm{~s}^{-1} \mathrm{sr}^{-1}\right)$.

was $27 \%$ greater in the $2.12 \mu \mathrm{m}$ image than in the $\mathrm{Brg} 45$ image. If we assume the $\mathrm{Brg} 45$ image is pure continuum and subtract that flux from the $2.12 \mu \mathrm{m}$ image, the remaining flux is $9.5 \times 10^{-5} \mathrm{ergs} \mathrm{cm}^{-2} \mathrm{~s}^{-1}$ for this knot; which can be considered a lower limit of flux from either $\mathrm{H}_{2}$, He I, or a combination of both species.

However, without spectroscopic analysis of the knots in the NIR, we are unable to determine the extent of emission from either species. While these data do not yet exist for NGC 2392, archival spectral data from Spitzer in $8-14 \mu \mathrm{m}$ band which includes the knot region of NGC 2392 does exist (PI James Houck, PID: 30482). In addition to the NIR ro-vibrational lines, $\mathrm{H}_{2}$ exhibits pure rotational lines in the mid-IR, namely the $0-0$ $\mathrm{S}(2) \lambda 12.28 \mu \mathrm{m}, 0-0 \mathrm{~S}(3) \lambda 9.665 \mu \mathrm{m}, \&$ 0-0 S(4) $\lambda 8.026 \mu \mathrm{m}$. Mata et al. (2016) has shown emission from these transitions can be seen in other $\mathrm{H}_{2}$ bearing PNe (e.g. NGC 2346, NGC $6720, \&$ NGC 7293). The data consists of 15 long slit positions covering roughly $2 / 3$ of the 


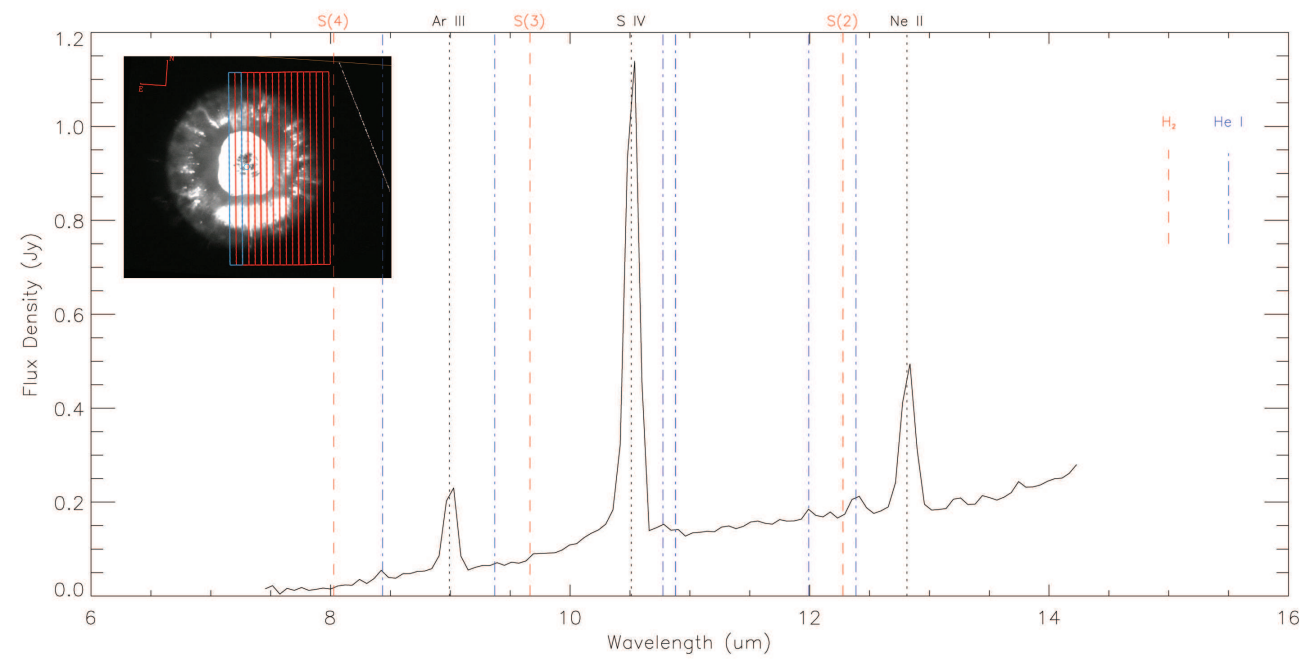

Figure 4.5: 8-12 $\mu \mathrm{m}$ spectrum of NGC 2392, Spitzer. Inset: Map of slit positions

nebula including the knots (Figure 4.5). The spectrum was summed over all slits and the result can be seen in Figure 4.5. The spectrum is dominated by the three lines: Ar III, S IV, and Ne II at $8.99 \mu \mathrm{m}, 10.51 \mu \mathrm{m}$ and $12.81 \mu \mathrm{m}$ respectively. Several other faint lines can be attributed to He I. There is no evidence for $\mathrm{H}_{2}$ emission seen in the spectrum.

\subsection{Discussion}

Despite measuring a higher flux from several knots in the $2.12 \mu \mathrm{m}$ image compared to the offset image, there is no definitive evidence of any $\mathrm{H}_{2}$ emission from NGC 2392 based on NIR narrow band imaging or mid-IR spectroscopy. Even if the excess flux in the knots proves to be from $\mathrm{H}_{2}, \mathrm{Br} \gamma$ emission is clearly dominant in the knots of NGC 2392 in contrast to the $\mathrm{H}_{2}$ dominant knots of the Helix nebula (Matsuura et al., 2009). We will therefore spend the remainder of the discussion addressing possible reasons for the lack of $\mathrm{H}_{2}$ emission in NGC 2392. 
The lack of $\mathrm{H}_{2}$ emission can be attributed to two broad scenarios: There is no $\mathrm{H}_{2}$ present in the nebula; or there is $\mathrm{H}_{2}$ present, but it is not excited to the degree necessary for detection. Due to abundant $\mathrm{H}$ lines, it is unlikely the nebula is $\mathrm{H}$ poor, therefore the lack of $\mathrm{H}_{2}$ may be attributable to a hindrance in formation. Molecular hydrogen formation requires high density, low temperatures, shielding from destructive UV photons, and typically dust grain surfaces as a catalyst (Hollenbach and Salpeter, 1971). During the late AGB phase, the high density circumstellar shell of dust and gas provides an environment very well suited for $\mathrm{H}_{2}$ formation. At the onset of the PN phase $\left(\mathrm{T}_{C S P N}>30,000 \mathrm{~K}\right)$, the increase in $\mathrm{UV}$ radiation destroys most of the $\mathrm{H}_{2}$, save for regions where the $\mathrm{H}_{2}$ can be shielded - such as cometary knots. Furthermore, it is hypothesized that cometary knots provide the environment necessary for continued $\mathrm{H}_{2}$ formation in addition to its survival (Speck et al., 2002, 2003). Knots are clearly present in NGC 2392, however their dust component could be lacking, inhibiting the formation of $\mathrm{H}_{2}$. A dust deficiency in the nebula is possible based on the lack of several spectroscopic dust features. Delgado-Inglada and Rodriguez (2014) found no evidence of dust features from PAH, SiC, Silicates, or a $30 \mu \mathrm{m}$ feature from the analysis of a Spitzer 5-35 $\mu \mathrm{m}$ spectrum. It is therefore possible that $\mathrm{H}_{2}$ formation is slow or not present in NGC 2392 due to being dust deficient. Furthermore, Casassus and Roche (2001) found weak or no warm dust features in the nebula in the 8-12 $\mu \mathrm{m}$ range, however, Spitzer spectra does indicate a dust continuum longward of $20 \mu \mathrm{m}$. It should be noted that dust deficiency does not necessarily correspond to there being no dust, simply lacking specific dust features. Therefore, while the nebula may be dust deficient, it is likely not completely devoid of dust — weakening this hypothesis.

An alternative, and more likely, mechanism to inhibit $\mathrm{H}_{2}$ formation is to invoke the presence of a sufficiently strong UV field during the AGB phase which would destroy any 
$\mathrm{H}_{2}$ typically formed during this evolutionary stage. The hot companion hypothesis, which attributes the presence of highly ionized atoms to a very hot companion star (see Section 2), could account for this UV field and could provide an explanation to several conspicuous properties of this nebula. Further study should seek out PNe which may provide further linkage between lack of $\mathrm{H}_{2}$ emission when accompanied by a hot companion in order to test this hypothesis.

The second scenario explaining lack of $\mathrm{H}_{2}$ emission in NGC 2392 is that there is $\mathrm{H}_{2}$ present (shielded within the knots), but it is not sufficiently excited and therefore unobserved. In $\mathrm{PNe}, \mathrm{H}_{2}$ can become excited to higher ro-vibrational states by either by UV pumping (fluorescence) or by shocks. Emission is then observed in the near and mid-IR as the $\mathrm{H}_{2}$ cascades back to the ground state. In the case of the Helix nebula, the spectrum of individual knots was obtained in the range of 2-5 $\mu \mathrm{m}$ which is populated by the 1-0 and 2-1 ro-vibrational lines (Matsuura et al., 2007). The observed line ratios could not be explained by the fluorescent excitation alone due to the available stellar UV flux. As a result, these observations favor either purely shock-excited $\mathrm{H}_{2}$ emission or perhaps a combination of both fluorescent and shock excitation. Assuming the knots in NGC 2392 are similar to those in the Helix, the apparent lack of $\mathrm{H}_{2}$ emission could arise from the lack of shock excitation. NGC 2392 is significantly younger than the Helix, it is therefore possible that the main shock front has not yet reached the knots, so we do not see any $\mathrm{H}_{2}$ emission. As stated in Section 2, expansion velocities of the inner bubble of the nebula are $120 \mathrm{~km} / \mathrm{s}$ greater than the knots García-Díaz et al. (2012), therefore we would expect the inner expanding bubble to eventually over take the knots, possibly giving rise to future shock excitation. This hypothesis also plays into possible explanations of knot formation.

Three possible mechanisms for the formation of knots in PNe are fragmentation from the 
switching off of a fast wind (Manchado et al., 2015; García-Segura et al., 2006) or Raleigh Taylor (RT) instabilities caused by the interaction between either the shock or ionization front (García-Segura et al., 1999). In our hypothesis, which assumes that the fast moving 'inner bubble' is representative of a fast wind, we must assume that the knots are due to RT instabilities from the ionization front instead of the shock front. This also precludes the fragmentation hypothesis since it depends on a depressurization of a fast wind. A fourth possibility is that the knots already existed prior to the PN phase.

\subsection{Conclusions}

Deep, narrow band images were obtained around $2 \mu \mathrm{m}$ and analysis of archival Mid-IR spectroscopy for NGC 2392 in search of any $\mathrm{H}_{2}$ emission from the knots or otherwise. Results of this experiment are similar to previous findings: there is no appreciable $\mathrm{H}_{2}$ emission in NGC 2392. While observations of $\mathrm{H}_{2}$ in PNe show a clear trend toward clumpy emission (e.g. (Manchado et al., 2015; Matsuura et al., 2009; Speck et al., 2002, 2003)) prompting a link between knots and $\mathrm{H}_{2}$, the reverse is untrue, as evidenced by NGC 2392. Namely, clumpiness in PNe does not necessarily indicate $\mathrm{H}_{2}$ emission. Furthermore, comparing the new and archival observations to hypotheses for knot formation tend to rule out RT instabilities at the shock front and favor RT instabilities at the ionization front. 


\section{Chapter 5}

\section{Summary and concluding remarks}

By studying $\mathrm{H}_{2}$ and its link to cometary knots in a larger sample of nebulae, we can begin to paint a larger picture of $\mathrm{H}_{2}$-bearing $\mathrm{PNe}$ and the properties therein. While the paradigm of a knot embedded torus of clumpy excited $\mathrm{H}_{2}$ fits three well studied objects (Helix, Ring, and NGC 2346), it fails to explain our two case studies (Dumbbell and NGC 2392). The Dumbbell, which does contain $\mathrm{H}_{2}$-bearing cometary knots, has a different morphological structure which may factor into its multiple "structures" which exhibit $\mathrm{H}_{2}$ excitation. NGC 2392, which has obvious cometary knots, has no $\mathrm{H}_{2}$ emission based on our findings. Therefore, any model which explores cometary knots formation and the role of molecular hydrogen must account for these two non-conformist nebulae (and possibly others). At the minimum, the differences of the Dumbbell nebula and NGC 2392 from previous studies of the Helix and Ring nebulae indicate a need to expand our rather small sample of such objects so that we may begin to understand the processes behind $\mathrm{H}_{2}$ formation, excitation, and its role in cometary knots of the PN as a whole. 


\section{Bibliography}

Aleman, I. and Gruenwald, R. (2004), 'Molecular Hydrogen in the Ionized Region of Planetary Nebulae', ApJ 607, 865.

Aleman, I. and Gruenwald, R. (2011), 'H2 infrared line emission from the ionized region of planetary nebulae', $A \& A \mathbf{5 2 8}, 74$.

Alexander, D. R. and Ferguson, J. W. (1994), 'Low-temperature Rosseland opacities', The Astrophysical Journal 437, 879-891.

Aller, L. (1954), Astrophysics - Nuclear Transformations, Stellar Interiors \& Nebulae, The Ronald Press Co.

Benedict, G., McArthur, B., Napiwotzki, R. et al. (2009), 'Astrometry with the Hubble Space Telescope: Trigonometric Parallaxes of Planetary Nebula Nuclei NGC 6853, NGC 7293, Abell 31, and DeHt 5', AJ 138, 1969-1984.

Blackman, E., Frank, A. and Welch, C. (2001), 'Magnetohydrodynamic Stellar and Disk Winds: Application to Planetary Nebulae', ApJ 546, 288-298.

Blocker, T. and Schonberner, D. (1991), 'A 7-solar-mass AGB model sequence not complying with the core mass-luminosity relation.', $A \& A$ 244, L43-L46. 
Boffin, H., B., M., T., R. et al. (2012), 'An Interacting Binary System Powers Precessing Outflows of an Evolved Star', Science 338, 773-775.

Bowen, G. (1988), 'Dynamical modeling of long-period variable star atmospheres', ApJ 329, 299-317.

Bowen, G. and Willson, L. (1991), 'From wind to superwind - The evolution of mass-loss rates for Mira models', ApJL 375, L53-L56.

Capriotti, E. (1971), 'The Formation of Condensations in Planetary Nebulae. I', ApJ 166, 563-572.

Casassus, S. and Roche, P. F. (2001), 'The Galactic disc distribution of planetary nebulae with warm dust emission features - II', MNRAS 320, 424.

Curtis, H. (1918), 'The Planetary Nebulae', Publ. Lick Obs. 13, 55-74.

Dabrowski, I. (1984), 'The Lyman and Werner bands of H2', Canadian Journal of Physics 62, 1639-1664.

Danehkar, A., Frew, D. J., Parker, Q. A. and De Marco, O. (2012), 'Photoionization Models of the Eskimo Nebula: Evidence for a Binary Central Star?', IAUS 282, 470-471.

De Marco, O. (2009), 'The Origin and Shaping of Planetary Nebulae: Putting the Binary Hypothesis to the Test', PASP 121, 316.

De Marco, O., Passy, J. and Frew, D. (2013), 'The binary fraction of planetary nebula central stars - I. A high-precision, I-band excess search', MNRAS 428, 2118.

Delgado-Inglada, G. and Rodriguez, M. (2014), 'C/O Abundance Ratios, Iron Depletions, and Infrared Dust Features in Galactic Planetary Nebulae', ApJ 784, 173-191. 
Dijkstra, C. and Speck, A. (2006a), 'Shaping Bipolar Planetary Nebulae: How Mass Loss Leads to Waistline Development', ApJ 651, 288-293.

Dijkstra, C. and Speck, A. K. (2006b), 'Shaping Bipolar Planetary Nebulae: How Mass Loss Leads to Waistline Development', ApJ 651, 288.

Dorfi, E. and Hofner, S. (1996), 'Non-spherical dust driven winds of slowly rotating AGB stars', A\&A 313, 605-610.

Dyson, J., Hartquist, T., Pettini, M. et al. (1989), 'Condensations in the planetary nebula NGC 7293 - an origin in circumstellar SiO maser spots?', MNRAS 241, 625-630.

Dyson, J., Pittard, J., Meaburn, J. et al. (2006), 'The tails in the Helix Nebula NGC 7293', $A \& A 457,561-567$.

Edgar, R., Nordhaus, J., Blackman, E. et al. (2008), 'The Formation of Crystalline Dust in AGB Winds from Binary-induced Spiral Shocks', ApJL 675, L101-L104.

Gail, H. and Sedlmayr, E. (1999), 'Mineral formation in stellar winds. I. Condensation sequence of silicate and iron grains in stationary oxygen rich outflows', $A \& A 347,594-$ 616.

García-Díaz, M., Gutierrez, L., Steffen, W., Lopez, J. A. and Beckman, J. (2015), 'Internal Proper Motions in the Eskimo Nebula', ApJ 798, 129-138.

García-Díaz, M., Lopez, J. A., Steffen, W. and Richer, M. G. (2012), ‘A Detailed Morphokinematic Model of the Eskimo, NGC 2392: A Unifying View with the Cat's Eye and Saturn Planetary Nebulae', ApJ 761, 172-183. 
García-Segura, G. (2005), 'The Structuring of Planetary Nebulae” MAGNETIC FIELDS IN THE UNIVERSE: From Laboratory and Stars to Primordial Structures', AIP Conference Proceedings 784, 226-238.

García-Segura, G., Langer, N., Rozyczka, M. and Franco, J. (1999), 'Shaping Bipolar and Elliptical Planetary Nebulae: Effects of Stellar Rotation, Photoionization Heating, and Magnetic Fields', ApJ 517, 767.

García-Segura, G., Lopez, J., Steffan, W. et al. (2006), 'The Dynamical Evolution of Planetary Nebulae after the Fast Wind', ApJ 646, L61.

Habing, H. (1996), 'Circumstellar envelopes and Asymptotic Giant Branch stars', $A \& A R v$ 7, 97-207.

Habing, H. and Olofsson, H. (2004), Asymptotic Giant Branch Stars, Astronomy and Astrophysics Library, Springer.

Hajian, A., Terzian, Y. and Bignell, C. (1995), 'Planetary Nebulae Expansion Distances. II. NGC 6572, NGC6210, NGC 3242, and NGC 2392', AJ 109, 2600.

Harpaz, A. and Soker, N. (1994), 'Evaporation of Brown Dwarfs in AGB Envelopes', MNRAS 270, 734-742.

Hofner, S. and Dorfi, E. (1997), 'Dust formation in winds of long-period variables. IV. Atmospheric dynamics and mass loss', A\&A 319, 648-654.

Hollenbach, D. and Salpeter, E. E. (1971), 'Surface Recombination of Hydrogen Molecules', ApJ 163, 155. 
Hora, J. L., Latter, W. B. and Lynne, K. (1999), 'Investigating the Near-Infrared Properties of Planetary Nebulae. II. Medium-Resolution Spectra', ApJs 124, 195.

Huggins, P., Foreville, T., Bachiller, R. et al. (2002), 'High-Resolution CO and H2 Molecular Line Imaging of a Cometary Globule in the Helix Nebula', ApJ 573, L55-L58.

Iben, I. and Renzini, A. (1983), 'Asymptotic giant branch evolution and beyond', ARA\&A 21, 271-342.

Jones, D., Mitchell, D., Lloyd, M. et al. (2012), 'The morphology and kinematics of the Fine Ring Nebula, planetary nebula Sp 1, and the shaping influence of its binary central star', MNRAS 420, 2271-2279.

Kahn, F. and West, K. (1985), 'Shapes of planetary nebulae', MNRAS 212, 837-850.

Kastner, J., Weintraub, D., Gatley, I. et al. (1996), 'H2 Emission from Planetary Nebulae: Signpost of Bipolar Structure', ApJ 462, 777-785.

Kudritzki, R., Mendez, R. H., Puls, J. and McCarthy, J. K. (1997), 'Winds in the atmospheres of central stars of Planetary Nebulae', IAUS 180, 64.

Kwok, S. (1982), 'From red giants to planetary nebulae', ApJ 258, 280-288.

Kwok, S. (2004), 'The synthesis of organic and inorganic compounds in evolved stars', Nature 430, 985-981.

Kwok, S., Purton, C. and Fitzgerald, P. (1978), 'On the origin of planetary nebulae', ApJL 219, L125-L127.

Latter, W., Dayal, A., Beiging, J. et al. (2000), 'Revealing the Photodissociation Region: HST/NICMOS Imaging of NGC 7027', ApJ 539, 783-797. 
Latter, W., Dayal, A., Bieging, J. et al. (2000), 'Revealing the Photodissociation Region: HST/NICMOS Imaging of NGC 7027', ApJ 539, 783.

Likkel, L., Dinerstein, H., Lester, D., Kindt, A. and Bartig, K. (2006), 'A Spectrophotometric Survey of K-Band Emission Lines in Planetary Nebulae', AJ 131, 1515.

Lodders, K. and Fegley, B. (1999), 'Condensation Chemistry of Circumstellar Grains', IAUS 191, 279.

Manchado, A., Stanghellini, L., Villaver, E., Garcia-Segura, G., Shaw, R. and GarcíaHernandez, D. A. (2015), 'High-resolution Imaging of NGC 2346 with GSAOI/GeMS: Disentangling the Planetary Nebula Molecular Structure to Understand Its Origin and Evolution', ApJ 808, 115.

Marquez-Lugo, R. A., Ramos-Larios, G., Guerrero, M. A. and Vazquez, R. (2013), 'On the relationship between the $\mathrm{H} 2$ emission and the physical structure of planetary nebulae', MNRAS 429, 973.

Mastrodemos, N. and Morris, M. (1998), 'Bipolar Preplanetary Nebulae: Hydrodynamics of Dusty Winds in Binary Systems. I. Formation of Accretion Disks', ApJ 497, 303.

Mata, H., Ramos-Larios, G., Guerrero, M. A. et al. (2016), 'Spitzer mid-infrared spectroscopic observations of planetary nebulae', MNRAS 459, 841 .

Matsuura, M., Speck, A., B., M. et al. (2009), 'A Firework of H2 Knots in the Planetary Nebula NGC 7293 (The Helix Nebula)', ApJ 700, 1067-1077.

Matsuura, M., Speck, A. and Smith, M. (2007), 'VLT/near-infrared integral field spectrometer observations of molecular hydrogen lines in the knots of the planetary nebula NGC 7293 (the Helix Nebula)', MNRAS 382, 1447-1459. 
Meaburn, J., Clayton, C., Bryce, M. et al. (1998), 'The nature of the cometary knots in the Helix planetary nebula (NGC 7293)', MNRAS 294, 201.

Meixner, M., McCullough, P., Harman, J. et al. (2005), 'The Multitude of Molecular Hydrogen Knots in the Helix Nebula', AJ 130, 1784-1794.

Meixner, M., Ueta, T., Dayal, A. et al. (1999), 'A Mid-infrared Imaging Survey of ProtoPlanetary Nebula Candidates', ApJS 122, 221-242.

Mellema, G., Raga, A., Canto, J. et al. (1998), 'Photo-evaporation of clumps in planetary nebulae', $A \& A$ 331, 335.

Natta, A. and Hollenbach, D. (1998), 'The evolution of the neutral gas in planetary nebulae: theoretical models', $A \& A$ 337, 517-538.

Nordhaus, J., Blackman, E. and Frank, A. (2007), 'Isolated versus common envelope dynamos in planetary nebula progenitors', MNRAS 376, 599-608.

O’Dell, C., Balick, B., Hajian, A. et al. (2002), 'Knots in Nearby Planetary Nebulae', AJ 123, 3329-3347.

O’Dell, C., Ferland, G., Henney, W. et al. (2013), 'Studies of NGC 6720 with Calibrated HST/WFC3 Emission-line Filter Images. I. Structure and Evolution', AJ 145, 92-111.

Oloffson, H. (2004), 'Highly episodic mass loss on the AGB: imaging in scattered stellar light', HST Proposal ID 10409.

Olofsson, H., Bergman, P., Lucas, R. et al. (2000), 'A high-resolution study of episodic mass loss from the carbon star TT Cygni', $A \& A$ 353, 583-597. 
onçalves, D., Corradi, R. and Mampaso, A. (2001), 'Low-Ionization Structures in Planetary Nebulae: Confronting Models with Observations', ApJ 547, 302-310.

Pauldrach, A., Hoffmann, T. L. and Mendez, R. H. (2004), 'Radiation-driven winds of hot luminous stars. XV. Constraints on the mass-luminosity relation of central stars of planetary nebulae', $A \& A \mathbf{4 1 9}, 1111$.

Persson, S., Murphy, D. C., Krzeminski, W., Roth, M. and Rieke, M. J. (1998), 'A New System of Faint Near-Infrared Standard Stars', AJ 116, 2475.

Pittard, J., Dyson, J., Falle, S. and Hartquist, T. (2005), 'Dynamical and pressure structures in winds with multiple embedded evaporating clumps - I. Two-dimensional numerical simulations', MNRAS 361, 1077-1090.

Pittard, J., Falle, S., Hartquist, T. and Dyson, J. (2009), 'The turbulent destruction of clouds - I. A $k-\varepsilon$ treatment of turbulence in 2D models of adiabatic shock-cloud interactions', MNRAS 394, 1351-1378.

Pottasch, S. R., Bernard-Salas, J. and Roellig, T. L. (2008), 'Abundances of planetary nebula NGC 2392', A\&A 481, 393.

Pottasch, S. R., Wesselius, P. R., Wu, C. et al. (1978), 'Ultraviolet radiation from planetary nebulae. II. Radiation from the Central Stars', $A \& A \mathbf{6 2}, 95$.

Redman, M., Viti, S., Cau, P. and Williams, D. (2003), 'Chemistry and clumpiness in planetary nebulae', MNRAS 345, 1291-1296.

Rola, C. and Stasińska, G. (1994), 'The carbon abundance problem in planetary nebulae', $A \& A$ 282, 199. 
Sahai, R. (2004), 'Sowing the Seeds of Asymmetry: Jet-like Outflows in Proto-Planetary Nebulae and AGB Stars', ASP Conference Proceedings 313, 141.

Sahai, R. (2009), 'Understanding Mass-Loss and the Late Evolution of Intermediate Mass Stars: Jets, Disks, Binarity, Dust, and Magnetic Fields', Astro2010: The Astronomy and Astrophysics Decadal Survey - Science White Papers 256, 0-0.

Smith, M., Davis, C. and Lioure, A. (1997), 'The ortho and para fractions of molecular hydrogen in protostellar outflows and Herbig-Haro objects', $A \& A$ 327, 1206-1214.

Soker, N. (1998), 'Radially Aligned Clumps and Tails in Planetary Nebulae', MNRAS 299, 861-866.

Soker, N. (2000), 'Dust formation and inhomogeneous mass-loss from asymptotic giant branch stars', MNRAS 312, 217.

Soker, N. and Subag, E. (2005), 'A Possible Hidden Population of Spherical Planetary Nebulae', AJ 130, 2717-2724.

Speck, A., Meixner, M., Fong, D. et al. (2002), 'Large-Scale Extended Emission around the Helix Nebula: Dust, Molecules, Atoms, and Ions', AJ 123, 346-361.

Speck, A., Meixner, M., Jacoby, G. and Knezek, P. (2003), 'Molecular Hydrogen in the Ring Nebula: Clumpy Photodissociation Regions', PASP 115, 170-177.

Stencel, R., Nuth, J., Little-Marenin, I. et al. (1990), 'The formation and annealing of circumstellar dust, as gauged by IRAS low-resolution spectra and the microwave maser chronology', ApJL 350, L45-L48. 
Tielens, A. (1990), 'Towards a circumstellar silicate mineralogy', From Miras to planetary nebulae: Which path for stellar evolution?; Proceedings of the International Colloquium pp. 186-200.

Turner, J., Kirby-Docken, K. and Dalgarno, A. (1977), ‘The Quadrupole Vibration-Rotation Transition Probabilities of Molecular Hydrogen', ApJS 35, 281.

Vassiliadis, E. and Wood, P. (1993), 'Evolution of low- and intermediate-mass stars to the end of the asymptotic giant branch with mass loss', ApJ 413, 641-657.

Visniac, E. (1994), 'Nonlinear instabilities in shock-bounded slabs', ApJ 428, 186-208.

Vivini, B., Natta, A., Marconi, A. et al. (1999), 'A near-infrared study of the planetary nebula NGC 2346', A\&A 342, 823-830.

Volk, K., Xiong, G. and Kwok, S. (2000), 'Infrared Space Observatory Spectroscopy of Extreme Carbon Stars', ApJ 530, 408-417.

Wang, W.-H. (2010), 'SIMPLE Imaging and Mosaicking PipeLinE', ASP Conference Series 434, 87.

Williams, R. (2003), 'Ionization Front Instabilities’, Rev. Mex. A\&A 15, 184-189.

Woitke, P. (2006), '2D models for dust-driven AGB star winds', $A \& A$ 452, 537-549.

Zanstra, H. (1955), 'On the formation of condensations in a gaseous nebula', Vistas in Astronomy 1, 256-268.

Zuckerman, B. and Aller, L. (1986), 'Origin of planetary nebulae - Morphology, carbonto-oxygen abundance ratios, and central star multiplicity', ApJ 301, 772-789. 
Zuckerman, B. and Gatley, I. (1988), 'Molecular hydrogen maps of extended planetary nebulae - The Dumbbell, the Ring, and NGC 2346', ApJ 324, 501-515. 


\section{VITA}

The author, Sean P. Baldridge, was born and grew up in Baton Rouge, Louisiana. Upon completion of high school, Sean enlisted in the United States Marine Corps as an electrician where he would spend the next 5 years. After his enlistment and two combat tours, he returned home to begin his career in physics. Sean graduated from Louisiana State University with a Bachelor of Science in physics with a concentration in astronomy on May 21, 2011. From there, he accepted an offer to attend graduate school at the University of Missouri. Under the tutelage of his adviser, Dr. Angela Speck, Sean chose to take on the research topic dealing with planetary nebulae using unpublished data of the Dumbbell nebula. Later, he obtained images of several other PN in search of $\mathrm{H}_{2}$ bearing cometary knots. After 6 years of research and teaching introductory astronomy, Sean obtained his PhD in Physics on December 15, 2017 from the University of Missouri. 